\title{
Optimal Multiresolution Quantization for Broadcast Channels with Random Index Assignment
}

\author{
Fei Teng
}

\author{
A thesis \\ presented to the University of Waterloo \\ in fulfillment of the \\ thesis requirement for the degree of \\ Master of Applied Science \\ in \\ Electrical and Computer Engineering
}

Waterloo, Ontario, Canada, 2010

(C) Fei Teng 2010 
I hereby declare that I am the sole author of this thesis. This is a true copy of the thesis, including any required final revisions, as accepted by my examiners.

I understand that my thesis may be made electronically available to the public. 


\begin{abstract}
Shannon's classical separation result holds only in the limit of infinite source code dimension and infinite channel code block length. In addition, Shannon theory does not address the design of good source codes when the probability of channel error is nonzero, which is inevitable for finite-length channel codes. Thus, for practical systems, a joint source and channel code design could improve performance for finite dimension source code and finite block length channel code, as well as complexity and delay.

Consider a multicast system over a broadcast channel, where different end users typically have different capacities. To support such user or capacity diversity, it is desirable to encode the source to be broadcasted into a scalable bit stream along which multiple resolutions of the source can be reconstructed progressively from left to right. Such source coding technique is called multiresolution source coding. In wireless communications, joint source channel coding (JSCC) has attracted wide attention due to its adaptivity to timevarying channels. However, there are few works on joint source channel coding for network multicast, especially for the optimal source coding over broadcast channels.

In this work, we aim at designing and analyzing the optimal multiresolution vector quantization (MRVQ) in conjunction with the subsequent broadcast channel over which the coded scalable bit stream would be transmitted. By adopting random index assignment (RIA) to link MRVQ for the source with superposition coding for the broadcast channel, we establish a closed-form formula of end-to-end distortion for a tandem system of MRVQ and a broadcast channel. From this formula we analyze the intrinsic structure of end-to-end distortion (EED) in a communication system and derive two necessary conditions for optimal multiresolution vector quantization over broadcast channels with random index assignment. According to the two necessary conditions, we propose a greedy iterative algorithm for jointly designed MRVQ with channel conditions, which depends on the channel only through several types of average channel error probabilities rather than the complete knowledge of the channel. Experiments show that MRVQ designed by the proposed algorithm significantly outperforms conventional MRVQ designed without channel information.

By building an closed-form formula for the weighted EED with RIA, it also makes the computational complexity incurred during the performance analysis feasible. In comparison with MRVQ design for a fixed index assignment, the computation complexity for quantization design is significantly reduced by using random index assignment. In addition, simulations indicate that our proposed algorithm shows better robustness against channel mismatch than MRVQ design with a fixed index assignment, simply due to the nature of using only the average channel information. Therefore, we conclude that our proposed algorithm is more appropriate in both wireless communications and applications where the complete knowledge of the channel is hard to obtain.
\end{abstract}


Furthermore, we propose two novel algorithms for MRVQ over broadcast channels. One aims to optimize the two corresponding quantizers at two layers alternatively and iteratively, and the other applies under the constraint that each encoding cell is convex and contains the reconstruction point. Finally, we analyze the asymptotic performance of weighted EED for the optimal joint MRVQ. The asymptotic result provides a theoretically achievable quantizer performance level and sheds light on the design of the optimal MRVQ over broadcast channel from a different aspect. 


\section{Acknowledgements}

I would like to first express my sincere gratitude to my thesis supervisor, Professor Enhui Yang, for his invaluable guidance and strong support throughout my M.A.Sc. program in University of Waterloo. I have tremendously benefited from his unique vision, technical insights and practical sensibility. I deeply appreciate his strict training and precious advice in all aspects of my academic development.

I thank my colleague Xiang Yu, for valuable discussions on research and precious friendship that support me throughout the past two years. Especially for my paper submitted to ISIT conference, his numerous proofreading and modification makes that paper possible.

I would like to thank my friends Lin, Jin, Yuhan, Zihan, Chang, Jie, Dake, Krzysztof for their company and support, and also thank my friend Shenghao, who is not here any more. I appreciate all the comfort from you when I feel horrible. I appreciate all the joy they have brought to me. I feel so blessed to have them on my side to unconditionally support me in my efforts towards my goals.

Finally, I wish to send my gratitude to my parents. Their unreserved love and support have been the most important power that kept my perseverance throughout the past years. Without their care and encouragement from a distance, I would not have been so focused in pursuing my study. The spirit of this thesis is dedicated to them. 


\section{Dedication}

To my parents, my girlfriend and all of my brothers. 


\section{Contents}

$\begin{array}{ll}\text { List of Tables } & \text { ix }\end{array}$

List of Figures $\quad$ xi

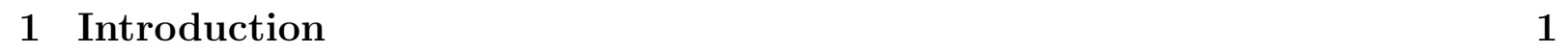

1.1 Multiresolution Quantization . . . . . . . . . . . . . . . . . 1

1.1.1 Single-resolution Vector Quantization . . . . . . . . . . . . . . . 1

1.1 .2 Multiresolution Vector Quantization . . . . . . . . . . . . . . . . 2

1.2 Joint Source-Channel Coding . . . . . . . . . . . . . . . . . . . 5

1.3 Index Assignment . . . . . . . . . . . . . . . . . . . . . . . 7

1.4 Motivation and Contribution . . . . . . . . . . . . . . . . . . 8

1.4.1 Theoretical side . . . . . . . . . . . . . . . . . . . 9

1.4 .2 Practical side . . . . . . . . . . . . . . . . 10

1.5 Organization of the thesis . . . . . . . . . . . . . . . . 10

2 Weighted End-To-End Distortion For A Tandem Source-Channel Coding System 11

$2.1 \quad$ System And Notation . . . . . . . . . . . . . . . . . . . . . . . . . 11

2.2 Multiresolution Modulation . . . . . . . . . . . . . . . . . 13

2.3 End-To-End Distortion With Random Index Assignment . . . . . . . . . . 15 
3 Joint Multiresolution Vector Quantization Design On Broadcast Channels 22

$3.1 \quad$ Optimal Design for Noisy Channel Multiresolution Quantization . . . . . . 22

3.2 Greedy Iterative Design Algorithm $\ldots \ldots \ldots \ldots$

3.3 Simulations . . . . . . . . . . . . . . . . . . . . . . . . . . . . . . . 29

3.4 "Empty Cells" Phenomenon . . . . . . . . . . . . . . . . . . . . 30

3.5 Comparison With The KR Algorithm . . . . . . . . . . . . . . . . . . 35

3.6 Novel Iterative Algorithms $\ldots \ldots \ldots \ldots$

$3.6 .1 \quad$ New iterative algorithm $\ldots \ldots \ldots \ldots$

3.6 .2 Controlled iterative algorithm $\ldots \ldots \ldots \ldots \ldots$

\begin{tabular}{|lll}
\hline 4 & The Asymptotic Minimum Weighted EED & 47
\end{tabular}

5 Conclusion and Future Work 51

5.1 Conclusion . . . . . . . . . . . . . . . . . . . . . . . . . . . . . . . 51

5.2 Future work $\ldots \ldots \ldots \ldots \ldots$

\begin{tabular}{ll}
\hline References & 52
\end{tabular} 


\section{List of Tables}

3.1 PSNR gain of our proposed MRVQ design over the separate MRVQ design for Gaussian source with zero mean and unit variance given $p_{e 1}=0.01$, $p_{e 2}=0.005$ and $p_{b}=0.02$. . . . . . . . . . . . . . . . . 30

3.2 Performance comparison in terms of PSNR (dB) for MRVQ designed by our proposed algorithm, the KR algorithm, and separate MRVQ design. . . . . 36

3.3 Sensitivity comparison in term of PSNR $(\mathrm{dB})$ for MRVQ designed by our proposed algorithm and the KR algorithm. . . . . . . . . . . . . . 37 


\section{List of Figures}

$1.1 \quad$ Diagram of lossy compression. . . . . . . . . . . . . . . . . . . . . 1

1.2 A four-resolution image description. . . . . . . . . . . . . . . . . . . . 3

1.3 A simple multicast system. . . . . . . . . . . . . . . . . . . . . . . . 8

2.1 Diagram of a tandem source and channel coding system with MRVQ and a broadcast channel. . . . . . . . . . . . . . . . 12

2.2 Superposition encoding scheme, where the constellations used by the two users are 16QAM and QPSK (a) enhanced signal $S_{e}(\mathrm{~b})$ base signal $S_{b}$ (c)

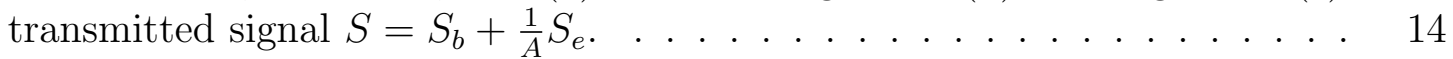

$3.1 \quad$ A typical set of $h_{i j}, i=1,2,3, j=1,2$. . . . . . . . . . . . . . . 26

$3.2 \quad$ Illustration of $z_{i j} \notin A_{i j}$ and empty cells, which causes divergence for MRVQ design by alternating (3.12), (3.13), (3.14). . . . . . . . . . 28

$3.3 \quad$ PSNR gain of channel optimized quantization over quantization designed separately from channels corresponding to various $p_{b}$ for a Gaussian source while fixing $p=0.5, p_{e 1}=0.01$ and $p_{e 2}=0.005$. . . . . . . . . . . . . 31

3.4 PSNR gain of channel optimized quantization over quantization designed separately from channels corresponding to various $p_{e 1}$ for a Gaussian source while fixing $p=0.5, p_{b}=0.02$ and $p_{e 2}=0.005$. . . . . . . . . . . . 32

3.5 PSNR gain of channel optimized quantization over quantization designed separately from channels corresponding to various $p_{e 2}$ for a Gaussian source while fixing $p=0.5, p_{b}=0.02$ and $p_{e 1}=0.01 \ldots \ldots . . \ldots 33$

3.6 Comparison between greedy iterative algorithm and new iterative algorithm corresponding to various $p_{b}$ for a Gaussian source while fixing $p=0.5$, $p_{e 1}=0.01$ and $p_{e 2}=0.005$. . . . . . . . . . . . . . . . . 40

3.7 Comparison between greedy iterative algorithm and new iterative algorithm corresponding to various $p_{e 1}$ for a Gaussian source while fixing $p=0.5$, $p_{b}=0.02$ and $p_{e 2}=0.005 \ldots \ldots \ldots \ldots$. . . . . . . . . . . 41 
3.8 Comparison between greedy iterative algorithm and controlled iterative algorithm corresponding to various $p_{b}$ for a Gaussian source while fixing $p=0.5, p_{e 1}=0.01$ and $p_{e 2}=0.005$. . . . . . . . . . . . . . . 45

3.9 Comparison between greedy iterative algorithm and controlled iterative algorithm corresponding to various $p_{e 1}$ for a Gaussian source while fixing $p=0.5, p_{b}=0.02$ and $p_{e 2}=0.005$. . . . . . . . . . . . . . . . 46 


\section{Chapter 1}

\section{Introduction}

\subsection{Multiresolution Quantization}

In a communication system, source coding for analog values is usually called quantization. As shown in Figure 1.1, a quantizer, by definition, maps the incoming sequence $Z_{1}, Z_{2}, Z_{2}, \cdots$, into a sequence of discrete random variables $\hat{Z}_{1}, \hat{Z}_{2}, \hat{Z}_{3}, \cdots$, where the objective is that $\hat{Z}_{i}$ should represent $Z_{i}$ with as little distortion as possible for each $i$. According to the number of resolutions, the lossy source coding could be categorized into single-resolution quantization and multiresolution quantization. Multiresolution quantization is some sort of extension for single-resolution quantization and widely used in multiuser systems, so first we will review the single-resolution case.

\subsubsection{Single-resolution Vector Quantization}

A source code designed to be used at a single rate and reproduction fidelity is a singleresolution source code, and vector quantization are block single-resolution source codes.

Theoretically, for a stationary source $Z$, given source alphabet $\mathcal{Z}$, reproduction alphabet $\hat{\mathcal{Z}}$ and distortion measure $d: \mathcal{Z} \times \hat{\mathcal{Z}} \longrightarrow[0, \infty)$, the rate distortion function $R(D)$ of the

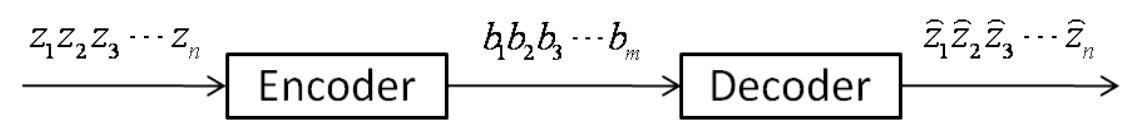

Figure 1.1: Diagram of lossy compression. 
source $Z$ with respect to $\mathcal{Z}, \hat{\mathcal{Z}}$ and $d$ is defined as

$$
R(D)=\inf _{\hat{Z}: d(Z ; \hat{Z}) \leq D} I(Z ; \hat{Z})
$$

where the infimum is taken over all possible random variables $\hat{Z}$ taking values in $\hat{\mathcal{Z}}$ and jointly distributed with $\mathrm{Z}$ such that $d(Z ; \hat{Z}) \leq D$

A n-dimensional vector quantizer $Q$ of level $L$ is a mapping from the n-dimensional Euclidean space $R^{n}$ on to a finite codebook $B$ :

$$
\boldsymbol{z}=z_{1} \cdots z_{n} \longrightarrow \hat{\boldsymbol{z}}=Q(\boldsymbol{z}) \in B=\boldsymbol{z}_{0}, \cdots, \boldsymbol{z}_{L-1}
$$

where $\boldsymbol{z}_{i} \in R^{n}, 0 \leq i \leq L-1$, are called codevectors or reproduction points.

Let $A_{i}=\left\{\boldsymbol{z} \in R^{n}: Q(\boldsymbol{z})=\boldsymbol{z}_{i}\right\}, 0 \leq i \leq L-1$. The set $A_{i}$ is called the cell associated with $z_{i}$. Given an n-dimensional random vector $Z^{n}=\left(Z_{1}, \cdots, Z_{n}\right)$ with joint pdf $p(\boldsymbol{z})=p\left(z_{1}, \cdot, z_{n}\right)$, the distortion resulting from quantization by $Q$ is

$$
D_{Q}=E_{Z^{n}}\left[d\left(Z^{n}, Q\left(Z^{n}\right)\right)\right],
$$

where

$$
d(\boldsymbol{z}, \hat{\boldsymbol{z}})=\frac{1}{n} \sum_{i=1}^{n}\left(z_{i}-\hat{z}_{i}\right)^{2}, \boldsymbol{z}=\left(z_{1}, \cdots, z_{n}\right), \hat{\boldsymbol{z}}=\left(\hat{z}_{1}, \cdots, \hat{z}_{n}\right)
$$

Note that a vector quantizer is uniquely determined by $\left\{A_{i}, \boldsymbol{z}_{i}\right\}_{i=0}^{L-1}$. Given a fixed coding dimension and distortion constraint $D$, an optimal vector quantizer is a vector quantizer that achieves $R^{n}(D)$, the lowest expected rate per symbol over all n-dimensional vector quantizers that require an expected distortion of no more than $D$ per sample. Since $R^{n}(D)$ converges to $R(D)$ as $n$ grows without bound, vector quantizers are asymptotically optimal single-resolution source codes. Equivalently, given fixed Lagrangian parameter $\lambda>0$, an optimal vector quantizer minimizes the Lagrangian performance $R+\lambda D$ over ndimensional vector quantizers. These optimality criteria form the basis of single-resolution vector quantizer design. Applying the alternating minimization procedure, we could design a fixed-rate or a variable-rate vector quantization.

\subsubsection{Multiresolution Vector Quantization}

A multiresolution source code (also known as successive refinement code) is a single compression system that describes data at a variety of rates and resolutions. A multiresolution source code creates a binary source description such that low-resolution descriptions are embedded in higher-resolution descriptions. Figure 1.2 demonstrates the action of a fourresolution source code. Decoding only the first $R_{1}$ bps yields a low-resolution reproduction 


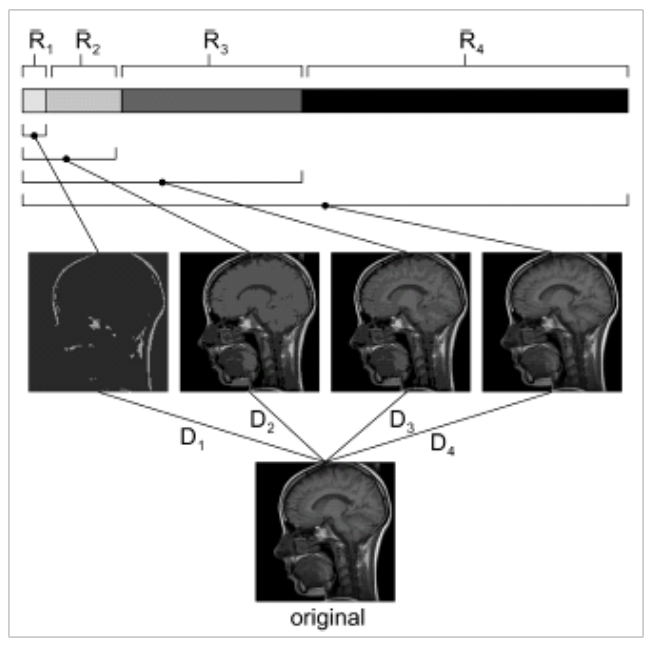

Figure 1.2: A four-resolution image description.

of the image with per sample distortion equal to $D_{1}$; decoding an additional $R_{2}$ bps (for a total description length of $R_{1}+R_{2}$ bps) yields a higher resolution source reproduction with per sample distortion $D_{1} \leq D_{2}$, and so on.

Multiresolution source coding is useful for applications where a single source must be described to a variety of different users or using an available rate that varies from system use to system use. For example, a single file on a particular web site may be examined by thousands of web site visitors. Some may want to see the data at high reproduction fidelity, others may favor fast transmission speed over reproduction quality, and still others may wish to determine acceptable reproduction quality during the data transfer process. Since single-resolution source codes fix a single rate and reproduction quality, no such code can satisfy all of these needs simultaneously. In contrast, a multiresolution source code yields a source description from which each user can decode to the rate or resolution most useful to him. Multiresolution codes are also useful in mobile communication systems, where the available communication rate may vary as a function of network traffic and physical location.

Multiresolution vector quantization (MRVQ) is block multiresolution source coding. In this case, the encoder blocks an incoming data stream into blocks of dimension $n$ and chooses for each n-block a collection of $n$-dimensional reproductions. In particular, in an $L$-resolution code, the encoder chooses $L$ reproductions for each data block. The encoder describes the chosen collection of reproductions using a fixed- or variable-rate binary string such that the first portion of the binary string describes the resolution-1 reproduction, the second portion of the binary string describes the resolution-2 reproduction given the resolution-1 reproduction already described, and so on. The decoder decodes the desired portion of the binary string, updating its source reproduction as the binary descriptions 
for higher and higher resolution reconstructions become available.

Multiresolution distortion-rate theory [2] addresses the question of multiresolution source code optimality in the limit of high coding dimension $n$. Given a stationary source, $\mathcal{R}_{n}^{f r, L}$ and $\mathcal{R}_{n}^{v r, L}$ are the closures of the set of rate-distortion pairs achievable on the source by fixed- and variable-rate n-dimension multiresolution vector quantizers, respectively. Let $\mathcal{R}_{n}^{L}$ denotes the corresponding information theoretic bound on the $L$-resolution rate-distortion region. Since $\mathcal{R}_{n}^{f r, L}$ and $\mathcal{R}_{n}^{v r, L}$ converge to $\mathcal{R}_{n}^{L}$, multiresolution vector quantizers are asymptotically optimal multiresolution source codes [2].

Given a fixed coding dimension $n$, an optimal fixed- or variable-rate multiresolution vector quantizer is an $n$-dimensional code that achieves rate-distortion performance on the lower boundary of $\mathcal{R}_{n}^{f r, L}$ or $\mathcal{R}_{n}^{v r, L}$. While $\mathcal{R}_{n}^{f r, L}$ and $\mathcal{R}_{n}^{v r, L}$ are not convex, Lagrangian methods may be used to find the lower convex hull of $\mathcal{R}_{n}^{f r, L}$ and $\mathcal{R}_{n}^{v r, L}$, given by

$$
\begin{aligned}
& j_{n}^{f r}\left(a^{L}, b^{L}\right)=\min _{\left(R^{L}, D^{L}\right) \in \mathcal{R}_{n}^{f r, L}} \sum_{l=1}^{L}\left[a_{l} D_{l}+b_{l} R_{l}\right] \\
& j_{n}^{v r}\left(a^{L}, b^{L}\right)=\min _{\left(R^{L}, D^{L}\right) \in \mathcal{R}_{n}^{v r, L}} \sum_{l=1}^{L}\left[a_{l} D_{l}+b_{l} R_{l}\right]
\end{aligned}
$$

Here, $\left(a^{L}, b^{L}\right)$ is described as the direction of a hyper-plane supporting the convex hull of the $L$-resolution operational rate-distortion region at a single point. It is general to use minimization of $\sum_{l=1}^{L}\left[a_{l} D_{l}+b_{l} R_{l}\right]$ as our optimality criterion for multiresolution vector quantizer design. Using this approach, a $n$-dimension fixed- or variable-rate vector quantizer is optimal if it lies on the lower convex hull of $\mathcal{R}_{n}^{f r, L}$ or $\mathcal{R}_{n}^{v r, L}$, respectively. This optimality criterion, first introduced in [5], can also be applied in other multiresolution codes.

In some related papers, and references therein, multiresolution source coding was mainly studied as a source coding technique independent of channel coding. For example, Effros et al. [2] developed some distortion-rate bounds for fixed- and variable-rate multiresolution vector quantization. In [4], criteria and algorithms for designing optimal MRVQ alone were investigated and developed. For discrete source with finite alphabet size, a globally optimal algorithm with lower complexity is illustrated in [3]. All these developments were made without reference to the subsequent channel over which the coded scalable bit stream would be transmitted. 


\section{$1.2 \quad$ Joint Source-Channel Coding}

Shannon's classical separation result states that we can optimize the end-to-end system design by separately optimizing source coding and channel coding [6]. However, this result holds only in the limit of infinite source code dimension and infinite channel code block length, and it does not provide a design algorithm for good channel codes with finite block length. In addition, Shannon theory does not address design of good source codes when the probability of channel error is nonzero, which is inevitable for finite-length channel codes. Thus, for practical systems, a joint source and channel code design could improve performance for finite dimension source code or finite block length channel code, as well as complexity and delay. Besides, joint source channel coding (JSCC) has attracted wide attention in wireless communications due to its adaptivity to time-varying channels. Previous work in the area of joint source and channel coding falls into three broad categories: source-optimized channel coding or modulation, channel-optimized source coding, and iterative algorithms, which combine these two code designs.

In source-optimized channel coding, the source code is designed for a noiseless channel. A channel code is then designed for this source code to minimize end-to-end distortion over the given channel, which is typically a binary symmetric channel (BSC), an additive white Gaussian noise (AWGN) channel with a given modulation, or a time-varying channel. Modestino and Daut provided an early treatment of source-optimized channel coding using differential pulse code modulation (DPCM) for image coding followed by convolutional channel coding [7]. They concluded that near optimal performance can be achieved using equal-error-protection channel codes. They also indicated that more flexible code rates are needed for source and channel code design, leading to subsequent work using ratecompatible punctured convolutional (RCPC) channel codes.

In source-optimized modulation, the source code is designed for a noiseless channel and then the modulation is optimized to minimize end-to-end distortion. Two papers address source-optimized modulation through an energy allocation strategy. In [8], a vector quantizer (VQ) is followed by multicarrier modulation, where the modulation provides unequal error protection to the different source bits by assigning different powers to each subcarrier. That work indicated that using modulation to obtain unequal error protection provides significant performance improvement.

Channel-optimized source coding is another approach for joint source channel coding. In this scenario, the source code maps the set of source symbols to binary strings for transmission over the channel. In fixed-rate source coding, the effect of a channel error is the receipt of an erroneous fixed-length binary string at the source decoder, which may cause the string to be decoded as an incorrect codeword. One way to achieve some degree of robustness to channel errors is to redesign the source codebook to take into account the index crossover probabilities imposed by a noisy channel. Examples of work taking this 
approach include the channel-optimized vector quantizer (COVQ) and its scalar variation [13], 14], [15]. COVQ is a vector quantizer that has been optimized for a given set of crossover probabilities of the source codeword indices [13], [14]. These codeword indices are generally mapped to binary strings, and the crossover probabilities are then functions of the channels bit error probability. The COVQ design involves iteratively optimizing source encoder and source decoder for the given set of index crossover probabilities. The result of this process is a locally optimal encoding rule for mapping source vectors to fixed-length binary indices and a corresponding decoding rule for mapping binary indices to their associated codewords, which may or may not be unique. Thus, inherent in the COVQ design algorithm is a block channel code design with hard-decision decoding.

Source-optimized channel coding and modulation can be combined with channel-optimized source coding using an iterative design. In [17, a variation of the generalized Lloyd algorithm is used to iteratively design a COVQ encoder, a modulation signal set, and a linear decoder. A similar approach for the joint design of a COVQ and multicarrier modulation appeared in [18. Neither of these approaches uses channel coding. In contrast, 12] used an iterative technique to jointly design a multistage VQ and trellis-coded modulation. In this work, the VQ is designed for a noiseless channel and then combined with index optimization. The effect of channel errors is not assumed negligible, so the bit allocation between the source and channel codes is implicitly chosen to force a small channel bit-error-probability.

A joint design of a source-optimized channel code, and a channel-optimized source code was proposed in [9]. The iterative joint code design uses COVQ for the source code and RCPC coding for the channel code. Ideally, the COVQ and the RCPC should be designed simultaneously. However, this simultaneous design is difficult to do in practice, leading to an iterative algorithm combining the design strategies of these two. The iterative design algorithm goes a step beyond, achieving a joint optimization of the source and channel codes using an iterative descent technique reminiscent of the generalized Lloyd algorithm [19] and guaranteeing convergence to a locally optimal code.

Source-optimized channel coding, channel-optimized source coding, and joint design of these two exhibit roughly the same distortion performance by choosing the optimal bit allocation between source coding and channel coding, shown in 9]. Obviously, the joint iterative design is much more complicated than the other two, so generally people would rather work on the other two. Most of the above works are written for the analysis of single-resolution source and channel code, and there are few works on joint source channel coding for broadcast networks.

In a related work, Kozintsev and Ramchandran [36] provided an algorithm for jointly designing multiresolution source quantization, multiresolution constellation, and the decoding strategy over time-varying channels, assuming a fixed index assignment and the complete knowledge of the channel. In practice, the complete channel information is dif- 
ficult to obtain and computation complexity is too high, so it is impractical to be implemented. However, as far as the author knows, it is the only work on channel optimized multiresolution quantization.

\subsection{Index Assignment}

One approach to improve the performance of a quantizer that transmits across a noisy channel is to design the quantizers encoder or decoder to specifically take into account the statistics of the transmission channel. Necessary optimality conditions for such channeloptimized encoders and decoders were given, for example, in [20], [14]. Alternatively, an explicit error control code can be cascaded with the quantizer, at the expense of added transmission rate. Additionally, the choice of index assignment in mapping source codewords to channel codewords can increase the performance of a quantization system with a noisy channel. Examples of index assignments include the natural binary code (NBC), the folded binary code, and the Gray code.

Ideally, one seeks a complete theoretical understanding of the structure and performance of a quantizer that transmits across a noisy channel, and whose encoder and decoder are channel optimized. Unfortunately, other than the optimality conditions given in [14], no other analytical results are known regarding such quantizers. Quantizer design and performance with index assignments for general encoders and decoders (i.e., not necessarily channel optimized) was considered in [21].

For uniform scalar quantizers with neither channel-optimized encoders nor decoders and with no explicit error control coding, formulas for the mean-squared error with uniform sources were given in [24] for the NBC, the Gray code, and for randomly chosen index assignments on a binary symmetric channel. They also asserted the optimality of the NBC for the binary symmetric channel. Crimmins et al. [25] proved the optimality of the NBC as asserted in [24], and McLaughlin, Neuhoff, and Ashley [28] generalized this result to uniform vector quantizers. Various other analytical results on index assignments without channel-optimized encoders or decoders have been given in [26], [27].

Quantizers with uniform encoders and channel-optimized decoders on binary symmetric channels were studied in [22]. For such quantizers, exact descriptions of the decoders were computed, and the asymptotic distributions of codevectors were determined for various index assignments. Distortions were calculated and compared to those of quantizers without channel optimization. The proof in [28] of the optimality of the NBC for quantizers with no channel optimization was extended in [22] to show that the NBC is also optimal for quantizers with uniform encoders and channel-optimized decoders. Quantizers with uniform decoders and channel-optimized encoders on over binary symmetric channels were studied in [23]. In that paper, the authors introduced a new affine index assignment called 


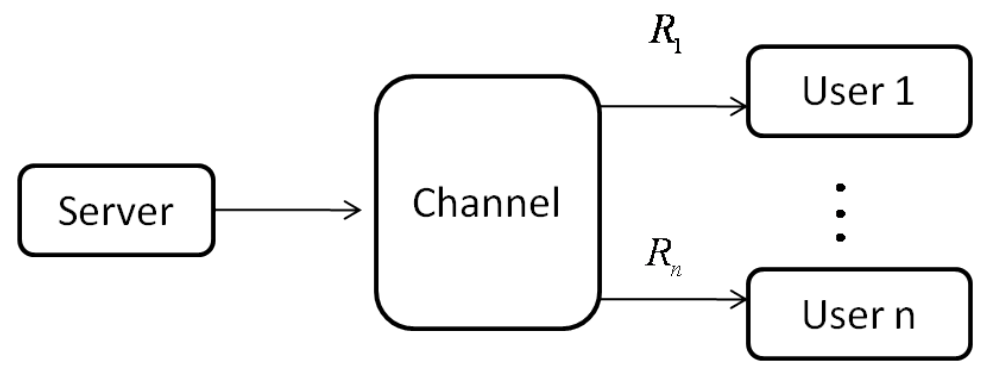

Figure 1.3: A simple multicast system.

the complemented natural code $(\mathrm{CNC})$, which turns out to have a number of interesting properties. It was shown that the NBC optimality does not extend to quantizers with uniform decoders and channel-optimized encoders. In fact, the CNC is shown to perform better than the NBC.

For more general cases, such as quantizers with channel-optimized encoders and decoders, no index assignment has been shown to be optimal. Though index assignment algorithms, such as binary switching, can choose a relatively good index assignment for the given vector quantization. However, the algorithms are guaranteed to converge to a locally optimal index assignment rather than the globally optimal one. Meanwhile an exhaustive approach to find the best index assignment is companied with too much complexity. Additionally, for a undesigned quantization system, how to choose a good index assignment is far from known. Especially for multiresolution quantization design, there is no such work on index assignments as far as the author knows.

Zeger and Manzella 32 defined a random index assignment to be mapping from codevectors to channel symbols chosen randomly and uniformly, and independent of the source, from the set of all possible permutations. It may be argued that this accurately models an arbitrary ordering of the codevectors in a codebook. In practice some implementations do in fact neglect to choose good index assignments and settle for whatever codebook ordering resulting from a quantizer design algorithm instead.

\subsection{Motivation and Contribution}

Consider a multicast system over a broadcast channel in Figure 1.3, where different end users typically have different capacities. To support such user or capacity diversity, it is desirable to encode the source to be broadcasted into a scalable bit stream along which multiple resolutions of the source can be reconstructed progressively from left to right.

Traditionally, source coding and channel coding are treated separately in such system, and the coding schemes are also optimized respectively. Since joint source channel coding 
has attracted wide attention in wireless communications due to its adaptivity to timevarying channels, it is a promising approach to improve the performance in a multiuser system. Therefore, in this thesis we focus on design of multiresolution quantization over broadcast channels by adopting joint source channel coding techniques and analyze the end-to-end performance of an entire system, which is really concerned by most people rather than the performance of pure source coding or channel coding.

\subsubsection{Theoretical side}

By using random index assignment (RIA) to link MRVQ for the source with superposition coding for the broadcast channel, we establish a closed-form formula of end-to-end distortion for a tandem system of MRVQ and a broadcast channel. Before this work there is no concise formula for predicting or closely bounding noisy broadcast channel quantizer distortion as far as author knows.

From the formula we analyze theoretically the intrinsic structure of EED in a communication system and derive two necessary conditions of an optimal multiresolution vector quantization over broadcast channels with random index assignment. The closed-form formula for weighed EED could be interpreted as the average of all possible index assignments given a set of transitional probabilities directly. On the other hand, it also refers to the average of all possible sets of transitional probabilities which subject to the same average error probabilities for any fixed index assignment. Thus, interestingly, this could also represent the performance for a class of channel conditions given a specific index assignment.

Moreover, the average distortion using a random index assignment gives us an analytic upper bound on the performance of the best possible index assignment. The performance of random index assignment in EED is usually close to that of the best index assignment, even when the best index assignment is significantly better than the average index assignment, their performances tend to follow the same trend as a function of the channel error probability. Another aspect of building a closed-form formula for the weighted EED is to make the computational complexity incurred during the performance analysis and quantization design feasible. In practice, naturally one would like to deal with the actual end-to-end distortion with the best index assignment. However, apart from the choose of index assignment, the computation complexity is a big issue. For example, we assume a 2-resolution quantization with $N_{1} \times N_{2}$ levels, which means there are $N_{1}$ levels for lowresolution quantization and each low-resolution description could be refined by another $\mathrm{N}_{2}$-level quantization. To design such quantization for a specific index assignment requires $\mathcal{O}\left(N_{1}^{2} N_{2}^{2}\right)$ arithmetic operations, as well as to calculate the actual end-to-end distortion, while our design or analysis consumes $\mathcal{O}\left(N_{1} N_{2}\right)$ with a random index assignment.

Our further efforts in this paper then reveal the asymptotic performance of the weighted EED for the optimal joint MRVQ. Though arbitrarily large number of quantization levels 
is not feasible for practical codes, the asymptotic result provides a theoretically achievable quantizer performance level and sheds light on the design of the optimal MRVQ.

\subsubsection{Practical side}

Based on two necessary conditions derived for minimizing the weighted EED, we propose an greedy iterative algorithm for jointly designing MRVQ with channel conditions. Experiment results show that the MRVQ designed by the proposed algorithm significantly outperforms conventional MRVQ designed without channel information.

To compare with the multiresolution vector quantization design for a fixed index assignment in [36] (hereafter referred to as the KR algorithm), we randomly generate several sets of channel transitional probabilities. What makes the comparison interesting is that the performance gain is marginal compared to the gain of the quantizers by our proposed algorithm over the quantizer designed by the separate quantization design. In addition, this marginal performance gain is achieved with much higher design complexity and with complete knowledge of all transitional probabilities. In practice, the complete channel information is difficult to obtain. Even if transitional probabilities are available, they are always fluctuating in wireless communications. In contrast, in our proposed algorithm, only certain types of symbol error probabilities of the broadcast channel are required. Simulations indicate that our proposed algorithm shows better robustness against the channel mismatch than the method with a fixed index assignment, simply by the nature of using the average channel information.

Additionally, we explore two novel algorithms for MRVQ design over broadcast channels. One aims to optimize high-resolution and low-resolution quantization alternatively and iteratively, and the other applies under the constraint that each encoding cell is convex and contains the reconstruction point. Experiments are conducted to compare our proposed algorithms from different aspects.

\subsection{Organization of the thesis}

The paper organization is as follows. First, we derive in Chapter II the closed-form formula for computing the EED of a tandem system with MRVQ, RIA, and a coded broadcast channel. Then, the algorithm design for optimal noisy channel quantization with random index assignment is discussed in Chapter III. Experimental and simulation results are also provided in Chapter III to illustrate the performance of our proposed iterative algorithms. The asymptotic analysis is included in Chapter IV, and finally Chapter V concludes the work. 


\section{Chapter 2}

\section{Weighted End-To-End Distortion For A Tandem Source-Channel Coding System}

In a quantization system that allows delay, the output binary data from a quantized sequence of input vectors can be blocked together and sent over the channel in the form of arbitrarily long channel codewords. Shannons channel coding theorem guarantees that up to $k C$ bits per source vector can be reliably conveyed in this way, hence achieving distortion down to $D(C)$, where $D(\cdot)$ is the distortion-rate function of the source and $C$ is the channel capacity. However, the same conclusion does not follow for source coding systems that do not allow delay; that is, systems that require that the $k R$ bits corresponding to an input vector be transmitted before the next input vector is encoded. Without arbitrarily long blocking of input symbols there exists a nonzero probability of incorrectly decoding a channel codeword. This in turn induces an extra component of distortion between the source vector and the final reproduction vector. Thus, in a communication system, it is meaningful to analyze the end-to-end distortion which consists of quantization distortion and that resulted from channel errors.

\subsection{System And Notation}

Let $\boldsymbol{z}$ be a k-dimensional real-valued vector source with a probability density function $f(\boldsymbol{z})$ over the k-dimensional Euclidean space $\Lambda$. Without loss of generality, we always assume that $\boldsymbol{z}$ has a zero mean. The variance of $\boldsymbol{z}$ is $\sigma^{2}=\frac{1}{k} \int_{\Lambda}\|\boldsymbol{z}\|^{2} f(\boldsymbol{z}) \mathrm{d} \boldsymbol{z}$.

Consider a tandem source-channel coding system as a concatenation of MRVQ and a coded broadcast channel, as shown in Figure 2.1. Without the loss of generality, we 


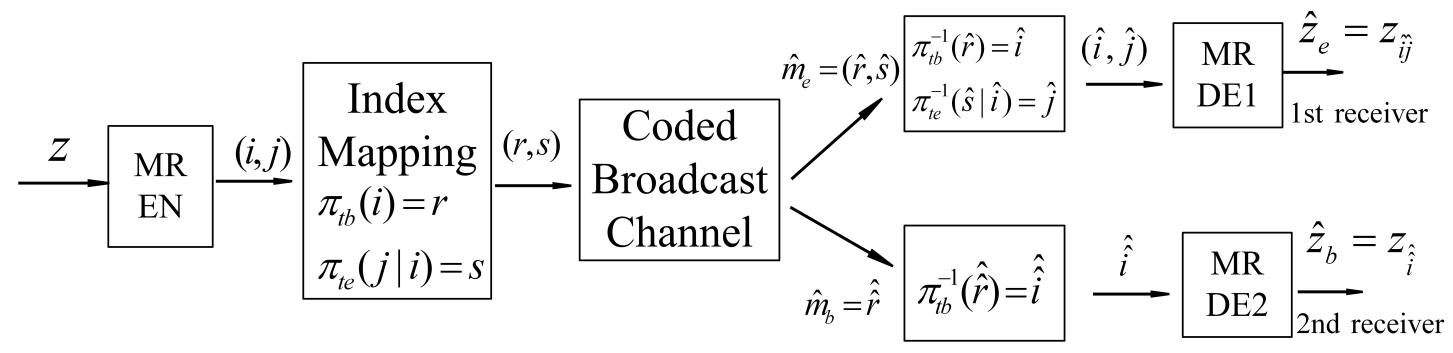

Figure 2.1: Diagram of a tandem source and channel coding system with MRVQ and a broadcast channel.

start with a two-resolution case, where the source is coded in two layers and two channels are used. In the low-resolution layer, the quantizer partitions the whole space $\Lambda$ into $N_{1}$ disjoint regions denoted as $\left\{A_{1}, \cdots, A_{N_{1}}\right\}$, and represents them with a set of code vectors $\left\{\boldsymbol{z}_{1}, \cdots, \boldsymbol{z}_{N_{1}}\right\}$ correspondingly. In the high-resolution layer, the quantizer further divides each $A_{i}$ into $N_{2}$ disjoint subregions denoted as $\left\{A_{i 1}, \cdots, A_{i N_{2}}\right\}$, and represents them with a set of code vectors $\left\{\boldsymbol{z}_{i 1}, \cdots, \boldsymbol{z}_{i N_{2}}\right\}$ for $N_{1} \geq i \geq 1$.

The two channels employ two different decoding strategies, i.e., the first channel assumes an output message set of $\boldsymbol{m}_{\mathrm{e}}=\left\{(r, s), r=1, \cdots, N_{1}, s=1, \cdots, N_{2}\right\}$, while the second channel assumes an output message set of $\boldsymbol{m}_{\mathrm{b}}=\left\{1, \cdots, N_{1}\right\}$. Then, the coded broadcast channel is characterized by a matrix of transitional probabilities,

$$
\mathbf{P}=\left\{p\left(\hat{m}_{\mathrm{e}}, \hat{m}_{\mathrm{b}} \mid(r, s)\right),(r, s) \in \boldsymbol{m}_{\mathrm{e}}, \hat{m}_{\mathrm{b}} \in \boldsymbol{m}_{\mathrm{b}}, \hat{m}_{\mathrm{e}} \in \boldsymbol{m}_{\mathrm{e}}\right\},
$$

where $p\left(\hat{m}_{\mathrm{e}}, \hat{m}_{\mathrm{b}} \mid(r, s)\right)$ means the probability that when message $(r, s)$ is transmitted, the first channel outputs $\hat{m}_{\mathrm{e}}$ and the second channel outputs $\hat{m}_{\mathrm{b}}$. From the matrix above, we could obtain another two matrixes of transitional probabilities

$$
\mathbf{P}_{\mathrm{e}}=\left\{p_{e}\left(\hat{m}_{\mathrm{e}} \mid(r, s)\right),(r, s) \in \boldsymbol{m}_{\mathrm{e}}, \hat{m}_{\mathrm{e}} \in \boldsymbol{m}_{\mathrm{e}}\right\}
$$

and

$$
\mathbf{P}_{\mathbf{b}}=\left\{p_{b}\left(\hat{m}_{\mathrm{b}} \mid(r, s)\right),(r, s) \in \boldsymbol{m}_{\mathrm{e}}, \hat{m}_{\mathrm{b}} \in \boldsymbol{m}_{\mathrm{b}}\right\} .
$$

for the first channel and the second channel respectively.

In the tandem system, we assume a one-to-one mapping $\pi_{t b}$ from $\left\{1, \cdots, N_{1}\right\}$ to $\left\{1, \cdots, N_{1}\right\}$, and a one-to-one mapping $\pi_{t e}(\cdot \mid i)$ from $\left\{1, \cdots, N_{2}\right\}$ to $\left\{1, \cdots, N_{2}\right\}$ for each $i=1, \cdots, N_{1}$. Denote a specific index assignment mapping as $\pi_{t}=\left(\pi_{t b}, \pi_{t e}\right)$, with $\left(\pi_{t b}(i), \pi_{t e}(j \mid i)\right)=(r, s),\left(\pi_{t b}(\hat{i}), \pi_{t e}(\hat{j} \mid \hat{i})\right)=(\hat{r}, \hat{s})$ and $\pi_{t b}(\hat{\hat{i}})=\hat{\hat{r}}$. The crossover error probabilities for source code vectors are related to channel transitional error probabilities as $p_{e}^{\pi_{t}}\left(\hat{\boldsymbol{z}}_{e}=\boldsymbol{z}_{\hat{i} \hat{j}} \mid \boldsymbol{z} \in A_{i j}\right)=p_{e}((\hat{r}, \hat{s}) \mid(r, s))$ which means the probability that the first receiver outputs $\boldsymbol{z}_{\hat{i} \hat{j}}$ given $\boldsymbol{z}$ in $A_{i j}$ is transmitted, and $p_{b}^{\pi_{t}}\left(\hat{\boldsymbol{z}}_{b}=\boldsymbol{z}_{\hat{\hat{i}}} \mid \boldsymbol{z} \in A_{i j}\right)=p_{b}(\hat{\hat{r}} \mid(r, s))$ which means the probability that the second receiver outputs $\boldsymbol{z}_{\hat{\hat{i}}}$ given $\boldsymbol{z}$ in $A_{i j}$ is transmitted. 
For simplicity, we denote $p_{e}^{\pi_{t}}\left(\hat{\boldsymbol{z}}_{e}=\boldsymbol{z}_{\hat{i} \hat{j}} \mid \boldsymbol{z} \in A_{i j}\right)$ and $p_{b}^{\pi_{t}}\left(\hat{\boldsymbol{z}}_{b}=\boldsymbol{z}_{\hat{\hat{i}}} \mid \boldsymbol{z} \in A_{i j}\right)$ as $p_{e}^{\pi_{t}}\left(\boldsymbol{z}_{\hat{i} \hat{j}} \mid \boldsymbol{z}_{i j}\right)$ and $p_{b}^{\pi_{t}}\left(\boldsymbol{z}_{\hat{\hat{i}}} \mid \boldsymbol{z}_{i j}\right)$ respectively. Given index assignment $\pi_{t}$, for the first receiver the EED is defined as

$$
D_{e}^{\pi_{t}} \triangleq \frac{1}{k} \sum_{i=1}^{N_{1}} \sum_{j=1}^{N_{2}} \int_{\boldsymbol{z} \in A_{i j}}\left[\sum_{\hat{i}=1}^{N_{1}} \sum_{\hat{j}=1}^{N_{2}}\left\|\boldsymbol{z}-\boldsymbol{z}_{\hat{i} \hat{j}}\right\|^{2} p_{e}^{\pi_{t}}\left(\boldsymbol{z}_{\hat{i} \hat{j}} \mid \boldsymbol{z}_{i j}\right)\right] f(\boldsymbol{z}) \mathrm{d} \boldsymbol{z}
$$

Similarly, for the second receiver, the EED is defined as

$$
D_{b}^{\pi_{t}} \triangleq \frac{1}{k} \sum_{i=1}^{N_{1}} \sum_{j=1}^{N_{2}} \int_{\boldsymbol{z} \in A_{i j}}\left[\sum_{\hat{i}=1}^{N_{1}}\left\|\boldsymbol{z}-\boldsymbol{z}_{\hat{i}}\right\|^{2} p_{b}^{\pi_{t}}\left(\boldsymbol{z}_{\hat{i}} \mid \boldsymbol{z}_{i j}\right)\right] f(\boldsymbol{z}) \mathrm{d} \boldsymbol{z}
$$

\subsection{Multiresolution Modulation}

Broadcast systems involve the transmission of information from a transmitter to many receivers. The information communicated to each user may be the same (e.g. TV broadcast) or it may be separate for each user (e.g. base station transmitting user-specific information). In current broadcast systems, the separation of user data is achieved by using orthogonal schemes in which the time and/or frequency is split between the users. Another transmission scheme for broadcast systems is to superimpose the user signals and use interference cancelation at the receiver. In this work we consider the case in which transmitter is sending common information to users, while each user could decode the data or resolution most useful to him according to the corresponding channel condition. As we know, superposition coding is an optimal choice when the broadcast channels are degraded, so we assume a superposition coding scheme instead of orthogonal schemes in this work.

In this case where a simple carrier broadcasting scheme over an AWGN channel is considered, a single transmitter attempts to send successive information to two or more receivers at the same time, where the information one user received is a part for the others. The capacity region for a two-receiver broadcast channel refers to the set of rate-pairs $\left(R_{1}, R_{2}\right)$ that can be achieved simultaneously with arbitrarily small probability of error [31].

Here we consider a simple broadcasting scheme, where the result transmitted signal $S$ is a linear superposition of the signals relative to the two users. Denoting $S_{b}$ the signal which corresponds to base information for low resolution reproduction and $S_{e}$ which corresponds 


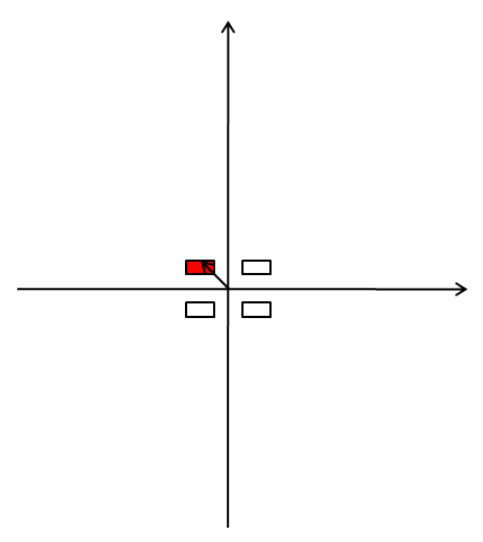

(a)

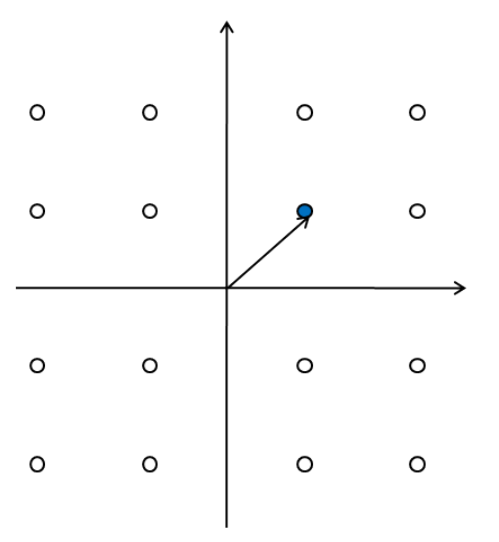

(b)

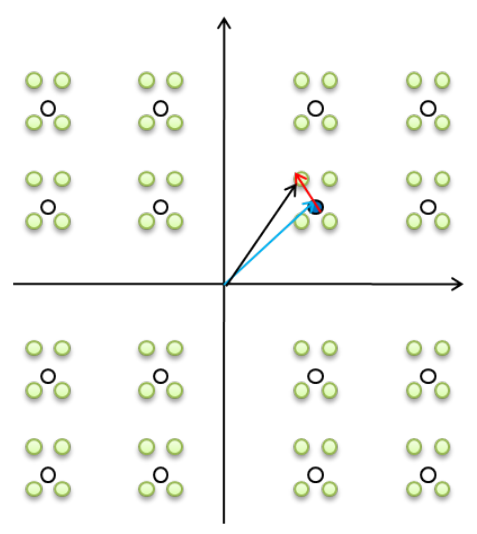

(c)

Figure 2.2: Superposition encoding scheme, where the constellations used by the two users are 16QAM and QPSK (a) enhanced signal $S_{e}$ (b) base signal $S_{b}$ (c) transmitted signal $S=S_{b}+\frac{1}{A} S_{e}$.

to the enhancement information for high resolution reproduction, the transmitted signal S is defined as follows:

$$
S=S_{b}+\frac{1}{A} S_{e}
$$

where $A$ is an attenuation factor which adjusts the power ratio between potential signals for base and enhancement information. In Figure 2.2, a superposition coding scheme is depicted, where $S_{b}$ is 16QAM and $S_{e}$ is QPSK signals and $A=8$.

One transmit antenna and one receive antenna are used. We denote $Y_{1}$ is the received signal at the first user side and $Y_{2}$ the received signal at the second user side in Figure 2.1. $Y_{1}$ and $Y_{2}$ can be expressed as follows:

$$
\begin{aligned}
& Y_{1}=h_{1} S+Z_{1} \\
& Y_{2}=h_{2} S+Z_{2}
\end{aligned}
$$

where $h_{1}$ and $h_{2}$ are respectively the channel gain of the first and second users. $Z_{1}$ and $Z_{2}$ are two additive white gaussian noises (AWGN) on the two channels having the same variance $N_{0}$ respectively. The second user treats the signal for enhancement information as a noise and decodes its data from $Y_{2}$, and the first user who has better channel condition performs a successive interference cancelation (SIC). That is, for the first user, after $S_{b}$ is decoded, it is stripped away from $Y_{e}$ to decode $S_{e}$ [30].

The capacity region of Gaussian broadcast channel with a single antenna at the transmitter and a single antenna at the receiver is known [31]. Splitting the total transmitted power $P$ in two parts: the power assigned to the base information denoted $P_{b}$ and the 
power assigned to the enhancement information denoted $P_{e}\left(P_{b}+P_{e}=P\right)$, the achievable rate pairs is given by [31]:

$$
\begin{aligned}
& R_{1}=\log _{2}\left(1+\frac{\left\|h_{1}\right\|^{2} P_{b}}{\left\|h_{1}\right\|^{2} P_{e}+N_{0}}\right)+\log _{2}\left(1+\frac{\left\|h_{1}\right\|^{2} P_{e}}{N_{0}}\right) \\
& R_{2}=\log _{2}\left(1+\frac{\left\|h_{2}\right\|^{2} P_{b}}{\left\|h_{2}\right\|^{2} P_{e}+N_{0}}\right)
\end{aligned}
$$

The choice of the ratio between $P_{b}$ and $P_{e}$, can control the two rates. Adaptive modulation can be used to adjust the constellation of the transmitted signal, in order to make second user decode $S_{b}$ with a very low probability of error. As known, Gaussian broadcast channel is degraded, and by using the superposition coding scheme, we could achieve the capacity when coding block length goes infinity [31] and transmit data most efficiently.

\subsection{End-To-End Distortion With Random Index As- signment}

Based on a superposition coding scheme, there exist $\left(N_{1} !\right)\left(N_{2} !\right)^{N_{1}}$ possible mappings from MRVQ codevectors to broadcast channel symbols. Assume a random selection of the index assignment with equal probability and define the average distortions for the first receiver and second receiver over all possible index assignments as $\bar{D}_{e}^{\Pi}$ and $\bar{D}_{b}^{\Pi}$ respectively. Thus, the closed-form formulas of these two average distortions are derived by the following theorem.

Theorem 1. For any $k$-dimensional multiresolution quantizer and coded broadcast channel as shown in Figure 2.1,

$$
D_{b}^{\Pi}=\left(1-\frac{N_{1} p_{b}}{N_{1}-1}\right) D_{Q_{b}}+\frac{N_{1} p_{b}}{N_{1}-1}\left(\sigma^{2}+S_{Q_{b}}\right)
$$

and

$$
D_{e}^{\Pi}=\left(1-p_{e 1}-\frac{N_{2} p_{e 2}}{N_{2}-1}\right) D_{Q_{e}}+\frac{N_{1} p_{e 1}}{N_{1}-1}\left(\sigma^{2}+S_{Q_{e}}\right)+\left(\frac{N_{2} p_{e 2}}{N_{2}-1}-\frac{p_{e 1}}{N_{1}-1}\right)\left[\bar{\sigma}_{Q_{e}}^{2}+\bar{S}_{Q_{e}}\right],
$$

where

$$
p_{b}=\frac{1}{N_{1}} \sum_{r=1}^{N_{1}} \frac{1}{N_{2}} \sum_{s=1}^{N_{2}} \sum_{\substack{k=1 \\ k \neq r}}^{N_{1}} p_{b}(k \mid(r, s))
$$




$$
\begin{gathered}
S_{Q_{b}} \triangleq\left(k N_{1}\right)^{-1} \sum_{i=1}^{N_{1}}\left\|z_{i}\right\|^{2} \\
D_{Q_{b}} \triangleq k^{-1} \sum_{i=1}^{N_{1}} \int_{z \in A_{i}}\left\|z-z_{i}\right\|^{2} f(z) \mathrm{d} z \\
p_{e 1}=\frac{1}{N_{1}} \sum_{r=1}^{N_{1}} \frac{1}{N_{2}} \sum_{s=1}^{N_{2}} \sum_{\substack{k=1 \\
k \neq r}}^{N_{1}} \sum_{l=1}^{N_{2}} p_{e}((k, l) \mid(r, s)) \\
p_{e 2}=\frac{1}{N_{1}} \sum_{r=1}^{N_{1}} \frac{1}{N_{2}} \sum_{s=1}^{N_{2}} \sum_{\substack{l=1 \\
l \neq s}}^{N_{2}} p_{e}((r, l) \mid(r, s)) \\
D_{Q_{e}} \triangleq k^{-1} \sum_{i=1}^{N_{1}} \sum_{j=1}^{N_{2}} \int_{z \in A_{i j}}\left\|z-z_{i j}\right\|^{2} f(z) \mathrm{d} z \\
S_{Q_{e}} \triangleq\left(k N_{1} N_{2}\right)^{-1} \sum_{i=1}^{N_{1}} \sum_{j=1}^{N_{2}}\left\|z_{i j}\right\|^{2}
\end{gathered}
$$

$\bar{S}_{Q_{e}} \triangleq \sum_{i=1}^{N_{1}} p\left(A_{i}\right)\left(\left(k N_{2}\right)^{-1} \sum_{\hat{j}=1}^{N_{2}}\left\|z_{i \hat{j}}-y_{i}\right\|^{2}\right)$ with $y_{i}$ being the conditional mean of $z$ given $A_{i}$ $\bar{\sigma}_{Q_{e}}^{2} \triangleq \sum_{i=1}^{N_{1}} p\left(A_{i}\right) \sigma_{i}^{2}$ with $\sigma_{i}^{2}$ being the conditional variance per dimension of $z$ given $A_{i}$.

Remark 1. In (2.5) and (2.6), $D_{Q_{b}}$ and $D_{Q_{e}}$ are the conventional quantization distortions at the lower and higher resolution layers, respectively; $S_{Q_{b}}$ and $S_{Q_{e}}$ are the scatter factors of the MRVQ at the lower and higher resolution layers, respectively, and represent respectively how far the codeword vectors at these two resolution layers are from the mean vector of the source. Similarly, $\bar{S}_{Q_{e}}$ can be interpreted as the average conditional scatter factor of the refinement coding given the partition $\left\{A_{i}\right\}$ at the lower resolution layer.

Remark 2. It is instructive to compare (2.6) with (2.5) in terms of the impact of channel error probabilities on the EED. In view of the definitions of $p_{e 1}$ and $p_{e 2}$, it follows that $p_{e 1}+p_{e 2}$ can be regarded as the average symbol error probability of the channel for the first receiver which tries to recover the higher resolution representation of the source. In comparison of (2.6) with (2.5), it is interesting and even surprising to see that it is $p_{e 1}$, not $p_{e 1}+p_{e 2}$, that appears before $\left(\sigma^{2}+S_{Q_{e}}\right)$ in (2.6). To certain degree, this implies that transmission errors occurring at the lower layer are more detrimental to the EED, a fact known more or less intuitively, but never quantified before. 
Proof. The proof of 2.6 is sketched as follows.

$$
\begin{aligned}
D_{e}^{\pi_{t}}= & D_{Q_{e}}+\frac{1}{k} \sum_{i=1}^{N_{1}} \sum_{j=1}^{N_{2}} \int_{\boldsymbol{z} \in A_{i j}}\left[\sum_{\substack{i=1 \\
i \neq i}}^{N_{1}} \sum_{\hat{j}=1}^{N_{2}}\left(2\left(\boldsymbol{z}-\boldsymbol{z}_{i j}\right)\left(\boldsymbol{z}_{i j}-\boldsymbol{z}_{\hat{i} \hat{j}}\right)^{\prime}+\left\|\boldsymbol{z}_{i j}-\boldsymbol{z}_{\hat{i} \hat{j}}\right\|^{2}\right) p_{e}^{\pi_{t}}\left(\boldsymbol{z}_{\hat{i} \hat{j}} \mid \boldsymbol{z}_{i j}\right)\right] f(\boldsymbol{z}) \mathrm{d} \boldsymbol{z} \\
& +\frac{1}{k} \sum_{i=1}^{N_{1}} \sum_{j=1}^{N_{2}} \int_{\boldsymbol{z} \in A_{i j}}\left[\sum_{\substack{\hat{j}=1 \\
j \neq j}}^{N_{2}}\left(2\left(\boldsymbol{z}-\boldsymbol{z}_{i j}\right)\left(\boldsymbol{z}_{i j}-\boldsymbol{z}_{i \hat{j}}\right)^{\prime}+\left\|\boldsymbol{z}_{i j}-\boldsymbol{z}_{i \hat{j}}\right\|^{2}\right) p_{e}^{\pi_{t}}\left(\boldsymbol{z}_{\hat{j}} \boldsymbol{z}_{i j}\right)\right] f(\boldsymbol{z}) \mathrm{d} \boldsymbol{z},
\end{aligned}
$$

where all vectors are row vectors and the transpose is indicated with a prime symbol. Then, take the average of the crossover error probabilities over all possible index assignment mappings. As to the first channel, we derive the crossover error probabilities,

a. For the first channel and for any two code vectors $\boldsymbol{z}_{i j}$ and $z_{\hat{i} \hat{j}}$ with different lowresolution layer indices, the crossover error probability is,

$$
\begin{aligned}
E_{\pi_{t}} p_{e}^{\pi_{t}}\left(\boldsymbol{z}_{\hat{i} \hat{j}} \mid \boldsymbol{z}_{i j}\right) & =E_{\pi_{t b}, \pi_{t e}} p_{e}\left(\left(\pi_{t b}(\hat{i}), \pi_{t e}(\hat{j} \mid \hat{i})\right) \mid\left(\pi_{t b}(i), \pi_{t e}(j \mid i)\right)\right) \\
& =\frac{1}{\left(N_{1} !\right)\left(N_{2} !\right)^{N_{1}}}\left(N_{1}-2\right) !\left[\left(N_{2}\right) !\right]^{\left(N_{1}-2\right)}\left[\left(N_{2}-1\right) !\right]^{2} \sum_{r=1}^{N_{1}} \sum_{\substack{k=1 \\
k \neq r}}^{N_{1}} \sum_{s=1}^{N_{2}} \sum_{l=1}^{N_{2}} p_{e}((k, l) \mid(r, s)) \\
& =\frac{1}{N_{2}\left(N_{1}-1\right)}\left[\frac{1}{N_{1} N_{2}} \sum_{r=1}^{N_{1}} \sum_{\substack{k=1 \\
k \neq r}}^{N_{1}} \sum_{s=1}^{N_{2}} \sum_{l=1}^{N_{2}} p_{e}((k, l) \mid(r, s))\right] \\
& =\frac{1}{N_{2}\left(N_{1}-1\right)} p_{e 1} .
\end{aligned}
$$

b. For the first channel and for any two code vectors $\boldsymbol{z}_{i j}$ and $z_{i j}$ with the same lowresolution layer index and different high-resolution layer indices, the crossover error probability is,

$$
\begin{aligned}
E_{\pi_{t}} p_{e}^{\pi_{t}}\left(\boldsymbol{z}_{i \hat{j}} \mid \boldsymbol{z}_{i j}\right) & =E_{\pi_{t b}, \pi_{t e}} p_{e}\left(\left(\pi_{t b}(i), \pi_{t e}(\hat{j} \mid i)\right) \mid\left(\pi_{t b}(i), \pi_{t e}(j \mid i)\right)\right) \\
& =\frac{1}{\left(N_{1} !\right)\left(N_{2} !\right)^{N_{1}}}\left(N_{1}-1\right) !\left[\left(N_{2}\right) !\right]^{\left(N_{1}-1\right)}\left(N_{2}-2\right) ! \sum_{r=1}^{N_{1}} \sum_{s=1}^{N_{2}} \sum_{\substack{l=1 \\
s \neq s}}^{N_{2}} p_{e}((r, l) \mid(r, s)) \\
& =\frac{1}{N_{1}} \sum_{r=1}^{N_{1}} \frac{1}{N_{2}} \sum_{s=1}^{N_{2}} \frac{1}{N_{2}-1} \sum_{\substack{l=1 \\
l \neq s}}^{N_{2}} p_{e}((r, l) \mid(r, s)) \\
& =\frac{1}{N_{2}-1} p_{e 2}
\end{aligned}
$$


Taking expectation over $D_{e}^{\pi_{t}}$ with respect to $\pi_{t}$ in (2.7) and substituting (2.8) and (2.9) in it leads to,

$$
\begin{aligned}
& D_{e}^{\Pi}=E_{\pi_{t}}\left(D_{e}^{\pi_{t}}\right)=E_{\pi_{t b}, \pi_{t e}}\left(D_{e}^{\pi_{t}}\right) \\
& =D_{Q_{e}}+\frac{p_{e 1}}{k\left(N_{1}-1\right) N_{2}} \sum_{i=1}^{N_{1}} \sum_{j=1}^{N_{2}} \int_{\boldsymbol{z} \in A_{i j}}\left[\sum_{\substack{i=1 \\
\hat{i} \neq i}}^{N_{1}} \sum_{\hat{j}=1}^{N_{2}}\left(2\left(\boldsymbol{z}-\boldsymbol{z}_{i j}\right)\left(\boldsymbol{z}_{i j}-z_{\hat{i} \hat{j}}\right)^{\prime}+\left\|z_{i j}-z_{\hat{i} \hat{j}}\right\|^{2}\right)\right] f(z) \mathrm{d} z \\
& +\frac{p_{e 2}}{k\left(N_{2}-1\right)} \sum_{i=1}^{N_{1}} \sum_{j=1}^{N_{2}} \int_{\boldsymbol{z} \in A_{i j}}\left[\sum_{\substack{j=1 \\
j \neq j}}^{N_{2}}\left(2\left(\boldsymbol{z}-\boldsymbol{z}_{i j}\right)\left(\boldsymbol{z}_{i j}-\boldsymbol{z}_{i \hat{j}}\right)^{\prime}+\left\|z_{i j}-z_{i \hat{j}}\right\|^{2}\right)\right] f(z) \mathrm{d} z \\
& =D_{Q_{e}}+\frac{p_{e 1}}{k\left(N_{1}-1\right) N_{2}} \sum_{i=1}^{N_{1}} \sum_{j=1}^{N_{2}} \int_{\boldsymbol{z} \in A_{i j}}\left[\sum_{\hat{i}=1}^{N_{1}} \sum_{\hat{j}=1}^{N_{2}}\left(2\left(\boldsymbol{z}-\boldsymbol{z}_{i j}\right)\left(\boldsymbol{z}_{i j}-z_{\hat{i} \hat{j}}\right)^{\prime}+\left\|z_{i j}-z_{\hat{i} \hat{j}}\right\|^{2}\right)\right] f(z) \mathrm{d} z \\
& +\left(\frac{p_{e 2}}{k\left(N_{2}-1\right)}-\frac{p_{e 1}}{k\left(N_{1}-1\right) N_{2}}\right) \sum_{i=1}^{N_{1}} \sum_{j=1}^{N_{2}} \int_{\boldsymbol{z} \in A_{i j}}\left[\sum_{\hat{j}=1}^{N_{2}}\left(2\left(\boldsymbol{z}-\boldsymbol{z}_{i j}\right)\left(z_{i j}-z_{i \hat{j}}\right)^{\prime}+\left\|z_{i j}-z_{i \hat{j}}\right\|^{2}\right)\right] f(z) \mathrm{d} z \\
& =D_{Q_{e}}+\frac{p_{e 1}}{k\left(N_{1}-1\right) N_{2}} \sum_{i=1}^{N_{1}} \sum_{j=1}^{N_{2}} \int_{z \in A_{i j}}\left[\sum_{\hat{i}=1}^{N_{1}} \sum_{\hat{j}=1}^{N_{2}}\left\|\boldsymbol{z}-\boldsymbol{z}_{\hat{i} \hat{j}}\right\|^{2}-N_{1} N_{2}\left\|\boldsymbol{z}-z_{i j}\right\|^{2}\right] f(z) \mathrm{d} z \\
& +\left(\frac{p_{e 2}}{k\left(N_{2}-1\right)}-\frac{p_{e 1}}{k\left(N_{1}-1\right) N_{2}}\right) \sum_{i=1}^{N_{1}} \sum_{j=1}^{N_{2}} \int_{\boldsymbol{z} \in A_{i j}}\left[\sum_{\hat{j}=1}^{N_{2}}\left\|\boldsymbol{z}-\boldsymbol{z}_{i \hat{j}}\right\|^{2}-N_{2}\left\|\boldsymbol{z}-z_{i j}\right\|^{2}\right] f(z) \mathrm{d} z \\
& =\left(1-\frac{N_{1} p_{e 1}}{N_{1}-1}\right) D_{Q_{e}}+\frac{p_{e 1}}{k\left(N_{1}-1\right) N_{2}} \sum_{\hat{i}=1}^{N_{1}} \sum_{\hat{j}=1}^{N_{2}} \int_{z \in \Lambda}\left\|z-z_{\hat{i} \hat{j}}\right\|^{2} f(z) \mathrm{d} z-\left(\frac{p_{e 2}}{N_{2}-1}-\frac{p_{e 1}}{\left(N_{1}-1\right) N_{2}}\right) N_{2} D_{Q_{e}} \\
& +\left(\frac{p_{e 2}}{k\left(N_{2}-1\right)}-\frac{p_{e 1}}{k\left(N_{1}-1\right) N_{2}}\right) \sum_{i=1}^{N_{1}} \sum_{j=1}^{N_{2}} \int_{\boldsymbol{z} \in A_{i j}} \sum_{\hat{j}=1}^{N_{2}}\left\|\boldsymbol{z}-\boldsymbol{z}_{i \hat{j}}\right\|^{2} f(\boldsymbol{z}) \mathrm{d} \boldsymbol{z} \\
& =\left(1-\frac{N_{2} p_{e 2}}{N_{2}-1}-p_{e 1}\right) D_{Q_{e}}+\frac{p_{e 1}}{\left(N_{1}-1\right) N_{2}}\left(N_{1} N_{2} \sigma^{2}+\frac{1}{k} \sum_{\hat{i}=1}^{N_{1}} \sum_{\hat{j}=1}^{N_{2}}\left\|\boldsymbol{z}_{\hat{i} \hat{j}}\right\|^{2}\right) \\
& +\left(\frac{p_{e 2}}{k\left(N_{2}-1\right)}-\frac{p_{e 1}}{k\left(N_{1}-1\right) N_{2}}\right) \sum_{i=1}^{N_{1}} \sum_{\hat{j}=1}^{N_{2}} \sum_{j=1}^{N_{2}} \int_{\boldsymbol{z} \in A_{i j}}\left\|\boldsymbol{z}-\boldsymbol{z}_{i \hat{j}}\right\|^{2} f(\boldsymbol{z}) \mathrm{d} \boldsymbol{z} \\
& =\left(1-\frac{N_{2} p_{e 2}}{N_{2}-1}-p_{e 1}\right) D_{Q_{e}}+\frac{p_{e 1}}{\left(N_{1}-1\right) N_{2}}\left(N_{1} N_{2} \sigma^{2}+S_{Q_{e}} N_{1} N_{2}\right) \\
& +\left(\frac{p_{e 2}}{k\left(N_{2}-1\right)}-\frac{p_{e 1}}{k\left(N_{1}-1\right) N_{2}}\right) \sum_{i=1}^{N_{1}} \sum_{\hat{j}=1}^{N_{2}} \int_{\boldsymbol{z} \in A_{i}}\left\|\boldsymbol{z}-\boldsymbol{z}_{i \hat{j}}\right\|^{2} f(\boldsymbol{z}) \mathrm{d} \boldsymbol{z}
\end{aligned}
$$




$$
\begin{aligned}
= & \left(1-\frac{N_{2} p_{e 2}}{N_{2}-1}-p_{e 1}\right) D_{Q_{e}}+\frac{p_{e 1}}{\left(N_{1}-1\right) N_{2}}\left(N_{1} N_{2} \sigma^{2}+S_{Q_{e}} N_{1} N_{2}\right) \\
& +\left(\frac{p_{e 2}}{N_{2}-1}-\frac{p_{e 1}}{\left(N_{1}-1\right) N_{2}}\right) \sum_{i=1}^{N_{1}} \sum_{\hat{j}=1}^{N_{2}} p\left(A_{i}\right)\left(\sigma_{i}^{2}+\frac{1}{k}\left\|\boldsymbol{z}_{i \hat{j}}-y_{i}\right\|^{2}\right) \\
= & \left(1-\frac{N_{2} p_{e 2}}{N_{2}-1}-p_{e 1}\right) D_{Q_{e}}+\frac{N_{1} p_{e 1}}{N_{1}-1} \sigma^{2}+\frac{N_{1} p_{e 1}}{N_{1}-1} S_{Q_{e}} \\
& +\left(\frac{p_{e 2}}{N_{2}-1}-\frac{p_{e 1}}{\left(N_{1}-1\right) N_{2}}\right)\left[N_{2} \bar{\sigma}_{Q_{e}}^{2}+N_{2} \sum_{i=1}^{N_{1}} p\left(A_{i}\right) S_{Q^{i}}\right] \\
= & \left(1-\frac{N_{2} p_{e 2}}{N_{2}-1}-p_{e 1}\right) D_{Q_{e}}+\frac{N_{1} p_{e 1}}{N_{1}-1} \sigma^{2}+\frac{N_{1} p_{e 1}}{N_{1}-1} S_{Q_{e}}+\left(\frac{N_{2} p_{e 2}}{N_{2}-1}-\frac{p_{e 1}}{N_{1}-1}\right)\left[\bar{\sigma}_{Q_{e}}^{2}+\bar{S}_{Q_{e}}\right] .
\end{aligned}
$$

The equality of 2.5 can be proved quite easily. According to coding scheme while $\boldsymbol{z}_{i j}$ is transmitted, the second receiver decodes it to $z_{\hat{\hat{i}}}$. Thus, $D_{b}^{\Pi}$ is a special case of $D_{e}^{\Pi}$ in terms of computation, where $z_{i j}=z_{i}$ for any $1 \leq i \leq N_{1}, 1 \leq j \leq N_{2}$. By using the conclusion in Theorem 1 , it is not hard to derive this formula.

Remark 3. From Theorem 1, it follows that the EED for high-quality reproduction is generated from five terms: quantization distortion, the joint impact of channel noise and the source variance, the joint term of channel noise and the scatter factor of the code vectors, the joint effect of channel noise and the average scatter factor of the code vectors and the joint effect of channel noise and the average conditional variance in the low-resolution layer. Intuitively, the more the code vectors scatter away from the source mean and away from the conditional mean of the corresponding partition of the low-resolution layer, the more contribution comes from the channel noise to the EED. Note that Theorem 1 provides a closed-form formula of average EED with high-quality reproduction for MRVQ on a broadcast channel with a random index assignment. Furthermore, it is valid for any MRVQ quantizer with either a high rate or a low rate.

Remark 4. From (2.5), it shows that EED for low-quality reproduction comes from three terms: quantization distortion, the joint impact of channel noise and the source variance, the joint term of channel noise and the scatter factor of the code vectors. Similar to high-quality case, the more the code vectors scatter away from the source mean, the more contribution comes from the channel noise to the EED.

Interestingly, the formula of the average EED for low-quality reproduction is the same as the result in the single resolution case [34]. If top-down approach is applied to design a tree-structured vector quantization, we could use the algorithm design in [34], and then the related analysis is still applicable. Note that top-down approaches sacrifice high-resolution performance for low-resolution performance and are thus most appropriate when focusing 
on the low-end user, who values the quality of the low-rate reproduction over the long term coding performance[4]. In this thesis, we will propose a different algorithm design by optimality later.

Corollary 1. Given a $k$-dimensional L-resolution quantizer with $\left\{A_{i_{1} \cdots i_{l}}, 1 \leq i_{j} \leq N_{j}, \forall 1 \leq\right.$ $j \leq l\},\left\{\mathbf{z}_{i_{1} \cdots i_{l}}, 1 \leq i_{j} \leq N_{j}, \forall 1 \leq j \leq l\right\}$ for the lth-resolution layer $(1 \leq l \leq L)$ and the corresponding noisy channel with transitional probability matrix $\left\{p_{l}\left(\left(\hat{r}_{1}, \cdots, \hat{r}_{l}\right) \mid\left(r_{1}, \cdots, r_{L}\right)\right)\right\}$, the average EED for the lth-resolution reproduction while transmitting a source $z$ with p.d.f $f(z)$ over a concatenation system with a random index assignment is

$$
D_{l}^{\Pi}=\left(1-\sum_{i=1}^{l-1} p_{l, i}-\frac{N_{l} p_{l, l}}{N_{l}-1}\right) D_{Q_{l}}+\sum_{i=1}^{l}\left(\frac{N_{i} p_{l, i}}{N_{i}-1}-\frac{p_{l, i-1}}{N_{i-1}-1}\right)\left(\left(\bar{\sigma}_{Q_{l}}^{(i-1)}\right)^{2}+\bar{S}_{Q_{l}}^{i-1}\right),
$$

where,

$$
\begin{aligned}
& p_{l, i}=\frac{1}{\prod_{j}^{L} N_{j}} \sum_{r_{1} \cdots r_{L}} \sum_{\substack{\hat{r}_{i}, \cdots, \hat{r}_{l} \\
r_{i} \neq r_{i}}} p_{l}\left(\left(r_{1}, \cdots, r_{i-1}, \hat{r}_{i}, \cdots, \hat{r}_{l}\right) \mid\left(r_{1}, \cdots, r_{L}\right)\right), \quad 1 \leq i \leq l \\
& D_{Q_{l}}=\frac{1}{k} \sum_{i_{1}, \cdots, i_{l}} \int_{\mathbf{z} \in A_{i_{1} \cdots i_{l}}}\left\|z-z_{i_{1} \cdots i_{l}}\right\|^{2} f(z) \mathrm{d} z, \\
& y_{i_{1} \cdots i_{m}}: \text { conditional mean of } \mathbf{z} \text { given } A_{i_{1} \cdots i_{m}}, \quad 1 \leq m \leq l-1 \\
& \sigma_{i_{1} \cdots i_{m}}^{2}: \text { conditional variance of } \mathbf{z} \text { given } A_{i_{1} \cdots i_{m}}, \quad 1 \leq m \leq l-1 \\
& S_{Q^{i_{1} \cdots i_{m}}}^{(l)}=\frac{1}{k \prod_{j=m+1}^{l} N_{j}} \sum_{\hat{i}_{m+1}, \cdots, \hat{i}_{l}}\left\|\mathbf{z}_{i_{1} \cdots i_{m} \hat{i}_{m+1} \cdots \hat{i}_{l}}-y_{i_{1} \cdots i_{m}}\right\|^{2}, \quad 1 \leq m \leq l-1 \\
& \left(\bar{\sigma}_{Q_{l}}^{(m)}\right)^{2}=\sum_{i_{1}, \cdots, i_{m}} p\left(A_{i_{1} \cdots i_{m}}\right) \sigma_{i_{1} \cdots i_{m}}^{2}, \quad 1 \leq m \leq l-1 \quad \text { the average conditional variance } \\
& \bar{S}_{Q_{l}}^{m}=\sum_{i_{1}, \cdots, i_{m}} p\left(A_{i_{1} \cdots i_{m}}\right) S_{Q^{i_{1} \cdots i_{m}}}^{(l)}, \quad 1 \leq m \leq l-1 \quad \text { average scatter factor } \\
& \bar{S}_{Q_{l}}^{0}=\frac{1}{k \prod_{j=1}^{l} N_{j}} \sum_{i_{i_{1}, \cdots, i_{l}}}\left\|\mathbf{z}_{i_{1} \cdots i_{l}}\right\|^{2}, \quad\left(\bar{\sigma}_{Q_{l}}^{(0)}\right)^{2}=\sigma^{2}, \quad p_{l, 0}=0 .
\end{aligned}
$$

This result could be derived by using similar process in Theorem 1. Details are omitted here.

Remark 5. Corollary 1 gives a closed-from formula of the average EED for the lthresolution reproduction among $L$ resolutions in total. Note that all the coefficients involving channel noise must sum to 1 . If combining all the terms involving $p_{l, i}$, the summation represents the distortion generated by wrong channel transmissions where error occurs at the ith layer and all the first $i-1$ layers are completely correct when using l-resolution reproduction. 
For some sources, the flexibility afforded by multiresolution source coding comes at a price. In particular, there exist some sources for which it is not possible to achieve the minimum distortion at each resolution simultaneously [1]. Thus, a optimality criterion, $\sum_{l=1}^{L}\left[a_{l} D_{l}+b_{l} R_{l}\right]$, is proposed in [4]. For a fixed-rate code, the optimality criterion is equivalent to the weighted criterion $\sum_{l=1}^{L} p_{l} D_{l}$ [37], with respect to distribution $\left\{p_{l}\right\}$ over the resolutions in a fixed-rate code. This same criterion, when extended to the vector $(k>1)$ case, allows the design of codes achieving any point on the convex hull of the closure of the set of rate-distortion vectors achievable by fixed-rate multiresolution quantizers [2]. Therefore, the optimality criterion in our scenario is defined as

$$
\bar{D}=p D_{e}^{\Pi}+(1-p) D_{b}^{\Pi},
$$

where $\mathrm{p}$ should be the distribution of the user with high-quality reproduction. This criterion represents the weighted EED in a two-user system.

From Theorem 1, it is now clear that minimizing $\left[p D_{Q b}+(1-p) D_{Q e}\right]$ alone does not necessarily reduce the weighted EED. To minimize the weighted EED, we should design a MRVQ so that for both two layers, their codevectors are spread widely enough to make $D_{Q e}, D_{Q b}$ and $\bar{\sigma}_{Q_{e}}^{2}$ small, and meanwhile are closely distributed around the source mean to make $S_{Q b}$ and $S_{Q e}$ also small. Besides, for the high-resolution layer, all the code vectors which are mapped to the same code vector in the low-resolution layer are concentrated around the conditional mean of the corresponding region in the low-resolution layer to reduce $\bar{S}_{Q_{e}}$. 


\section{Chapter 3}

\section{Joint Multiresolution Vector Quantization Design On Broadcast Channels}

In this section, we propose several algorithms for designing a generally optimal multiresolution vector quantizer on a broadcast channel with a random index assignment.

\subsection{Optimal Design for Noisy Channel Multiresolu- tion Quantization}

Similar to the development of algorithm for multiresolution vector quantization design without channel information, the optimal design for MRVQ on a broadcast channel is formulated as a minimization problem, i.e.,

$$
\min _{\mathfrak{Z}_{1}, \mathfrak{Z}_{2}} \min _{\mathfrak{A}} p D_{e}^{\Pi}+(1-p) D_{b}^{\Pi}
$$

where,

$\mathfrak{A}=\left\{A_{i j}, 1 \leq i \leq N_{1}, 1 \leq j \leq N_{2}\right\}$ with $\bigcup_{i, j} A_{i j}=\Lambda, \bigcup_{j} A_{i j}=A_{i}$ and $\int_{A_{i j} \cap A_{i^{\prime} j^{\prime}}} f(\boldsymbol{z}) \mathrm{d} \boldsymbol{z}=$ $0, \quad \forall(i, j) \neq\left(i^{\prime}, j^{\prime}\right)$,

$\mathfrak{Z}_{\mathbf{1}}=\left\{\boldsymbol{z}_{i}, i=1, \cdots, N_{1}\right\}$ is associated with a given partition $\left\{A_{i}, 1 \leq i \leq N_{1},\right\}$,

$\mathfrak{Z}_{2}=\left\{\boldsymbol{z}_{i j}, i=1, \cdots, N_{1}, j=1, \cdots, N_{2}\right\}$ is associated with a given partition $\left\{A_{i j}, 1 \leq i \leq\right.$ $\left.N_{1}, 1 \leq j \leq N_{2}\right\}$.

Based on Theorem 1, the following two necessary conditions can be derived for the optimal solution to 3.1 . 
Theorem 2. Given a coded broadcast channel with the transmission probability $p\left(\hat{m}_{e}, \hat{m}_{b} \mid(r, s)\right)$, the optimal multiresolution vector quantizer with a random index assignment satisfies the following two conditions.

1) For $\left\{A_{i j}, 1 \leq i \leq N_{1}, 1 \leq j \leq N_{2}\right\}$, the optimal code vectors by minimizing $\bar{D}$ in each layer are computed respectively by

$$
\begin{gathered}
\mathbf{z}_{i}=\frac{\int_{A_{i}} z f(\mathbf{z}) \mathrm{d} \mathbf{z}}{\frac{p_{b}}{N_{1}-1-N_{1} p_{b}}+p\left(A_{i}\right)}, \quad i=1, \cdots, N_{1} \\
\mathbf{z}_{i j}=\frac{k_{1} \int_{A_{i j}} z f(\mathbf{z}) \mathrm{d} \mathbf{z}+k_{2} \int_{A_{i}} z f(\mathbf{z}) \mathrm{d} \mathbf{z}}{k_{1} p\left(A_{i j}\right)+\frac{p_{e 1}}{N_{2}\left(N_{1}-1\right)}+k_{2} p\left(A_{i}\right)}, \quad i=1, \cdots, N_{1}, j=1, \cdots, N_{2}
\end{gathered}
$$

2) For $\left\{\mathbf{z}_{i}, i=1, \cdots, N_{1}\right\},\left\{\mathbf{z}_{i j}, i=1, \cdots, N_{1}, j=1, \cdots, N_{2}\right\}$, the optimal finest partition is achieved by

$$
\begin{array}{r}
A_{i j}=\left\{\mathbf{z}: 2 \alpha_{i j} \cdot(\mathbf{z})^{\prime}-\beta_{i j} \geq 2 \alpha_{i^{\prime} j^{\prime}} \cdot(\mathbf{z})^{\prime}-\beta_{i^{\prime} j^{\prime}}, \forall\left(i^{\prime}, j^{\prime}\right) \neq(i, j)\right\}, \\
i=1, \cdots, N_{1}, j=1, \cdots, N_{2}
\end{array}
$$

where,

$$
\begin{aligned}
& k_{1} \triangleq 1-p_{e 1}-\frac{N_{2} p_{e 2}}{N_{2}-1} \\
& k_{2} \triangleq \frac{p_{e 2}}{N_{2}-1}-\frac{p_{e 1}}{N_{2}\left(N_{1}-1\right)} \\
& k_{3} \triangleq 1-\frac{N_{1} p_{b}}{N_{1}-1} \\
& \alpha_{i j}=p k_{1} \mathbf{z}_{i j}+p k_{2} \sum_{j=1}^{N_{2}} \mathbf{z}_{i j}+(1-p) k_{3} \mathbf{z}_{i} \\
& \beta_{i j}=p k_{1} \mathbf{z}_{i j}^{2}+p k_{2} \sum_{j=1}^{N_{2}} \mathbf{z}_{i j}^{2}+(1-p) k_{3} \mathbf{z}_{i}^{2} .
\end{aligned}
$$

Proof. For 1$)$, given $\left\{A_{i j}, 1 \leq i \leq N_{1}, 1 \leq j \leq N_{2}\right\}$ the proof is a standard procedure to take the derivative of the objective function (7) over $\boldsymbol{z}_{i}$ and $\boldsymbol{z}_{i j}$ respectively. Specifically,

$$
\begin{aligned}
\frac{\partial \bar{D}}{\partial \boldsymbol{z}_{i}} & =\left(1-\frac{N_{1} p_{b}}{N 1-1}\right) \frac{\partial D_{Q_{b}}}{\partial \boldsymbol{z}_{i}}+\frac{N_{1} p_{b}}{N 1-1} \frac{\partial S_{Q_{b}}}{\partial \boldsymbol{z}_{i}} \\
& =\left(1-\frac{N_{1} p_{b}}{N 1-1}\right) \frac{\partial}{\partial \boldsymbol{z}_{i}}\left(\sigma^{2}-\frac{2}{k} \sum_{j=1}^{N_{1}} \int_{\boldsymbol{z} \in A_{j}} z f(\boldsymbol{z}) \mathrm{d} \boldsymbol{z} \cdot \boldsymbol{z}_{j}^{\prime}+\frac{1}{k} \sum_{j=1}^{N_{1}}\left\|\boldsymbol{z}_{j}\right\|^{2} p\left(\boldsymbol{z} \in A_{j}\right)\right)+2 \boldsymbol{z}_{i} \frac{1}{k} \frac{p_{b}}{N_{1}-1} \\
& =\left(1-\frac{N_{1} p_{b}}{N 1-1}\right) \frac{1}{k}\left(-2 \int_{\boldsymbol{z} \in A_{i}} z f(\boldsymbol{z}) \mathrm{d} \boldsymbol{z}+2 \boldsymbol{z}_{i} \cdot p\left(\boldsymbol{z} \in A_{i}\right)\right)+\frac{2}{k} \boldsymbol{z}_{i} \frac{p_{b}}{N_{1}-1} .
\end{aligned}
$$


Letting $\frac{\partial \bar{D}}{\partial \boldsymbol{z}_{i}}=0$ in the above leads to 3.2 .

$$
\begin{aligned}
\frac{\partial \bar{D}}{\partial \boldsymbol{z}_{i j}}= & \left(1-p_{e 1}-\frac{N_{2} p_{e 2}}{N_{2}-1}\right) \frac{\partial D_{Q_{e}}}{\partial \boldsymbol{z}_{i j}}+\frac{p_{e 1}}{N_{2}(N 1-1)} \frac{1}{k} 2 \boldsymbol{z}_{i j}+\left(\frac{p_{e 2}}{N_{2}-1}-\frac{p_{e 1}}{N_{2}\left(N_{1}-1\right)}\right) \int_{A_{i}} 2\left(\boldsymbol{z}_{i j}-\boldsymbol{z}\right) f(\boldsymbol{z}) \mathrm{d} \boldsymbol{z} \\
= & \left(1-p_{e 1}-\frac{N_{2} p_{e 2}}{N_{2}-1}\right) \frac{\partial}{\partial \boldsymbol{z}_{i j}}\left(\sigma^{2}-\frac{2}{k} \sum_{\hat{i}=1}^{N_{1}} \sum_{\hat{j}=1}^{N_{2}} \int_{\boldsymbol{z} \in A_{\hat{i} \hat{j}}} z f(\boldsymbol{z}) \mathrm{d} \boldsymbol{z} \cdot \boldsymbol{z}_{\hat{i} \hat{j}}^{\prime}+\frac{1}{k} \sum_{\hat{i}=1}^{N_{1}} \sum_{\hat{j}=1}^{N_{2}}\left\|\boldsymbol{z}_{\hat{i} \hat{j}}\right\|^{2} p\left(\boldsymbol{z} \in A_{\hat{i} \hat{j}}\right)\right) \\
& +\frac{p_{e 1}}{N_{2}(N 1-1)} \frac{1}{k} 2 \boldsymbol{z}_{i j}+\left(\frac{p_{e 2}}{N_{2}-1}-\frac{p_{e 1}}{N_{2}\left(N_{1}-1\right)}\right) \int_{A_{i}} 2\left(\boldsymbol{z}_{i j}-\boldsymbol{z}\right) f(\boldsymbol{z}) \mathrm{d} \boldsymbol{z} \\
= & \left(1-p_{e 1}-\frac{N_{2} p_{e 2}}{N_{2}-1}\right)\left(-2 \int_{\boldsymbol{z} \in A_{i j}} z f(\boldsymbol{z}) \mathrm{d} \boldsymbol{z}+2 \boldsymbol{z}_{i j} \cdot p\left(\boldsymbol{z} \in A_{i j}\right)\right)+\frac{p_{e 1}}{N_{2}(N 1-1)} \frac{1}{k} 2 \boldsymbol{z}_{i j} \\
& +\left(\frac{p_{e 2}}{N_{2}-1}-\frac{p_{e 1}}{N_{2}\left(N_{1}-1\right)}\right) \int_{A_{i}} 2\left(\boldsymbol{z}_{i j}-\boldsymbol{z}\right) f(\boldsymbol{z}) \mathrm{d} \boldsymbol{z}
\end{aligned}
$$

Letting $\frac{\partial \bar{D}}{\partial \boldsymbol{z}_{i j}}=0$ in the above leads to 3.3 .

For 2), applying the similar idea to the approaches in [37], [4] which is for multiresolution quantization design without reference to the channel noise, then yields the condition (3.4).

Given $\mathfrak{Z}_{1}$ and $\mathfrak{Z}_{2}$, the objective function can be written in integral form as

$$
\bar{D}=\int_{\Lambda} G(\boldsymbol{z}) f(\boldsymbol{z}) \mathrm{d} \boldsymbol{z}+\text { const }
$$

where,

$$
\text { if } \boldsymbol{z} \in A_{i j} \quad G(\boldsymbol{z})=F\left(\boldsymbol{z}, \boldsymbol{z}_{i}, \boldsymbol{z}_{i j}\right) \text {. }
$$

where,

$$
F\left(\boldsymbol{z}, \boldsymbol{z}_{i}, \boldsymbol{z}_{i j}\right) \triangleq \frac{p}{k}\left(k_{1}\left\|\boldsymbol{z}-\boldsymbol{z}_{i j}\right\|^{2}+k_{2} \sum_{\hat{j}=1}^{N_{2}}\left\|\boldsymbol{z}-\boldsymbol{z}_{i \hat{j}}\right\|^{2}\right)+\frac{(1-p) k_{3}}{k}\left\|\boldsymbol{z}-\boldsymbol{z}_{i}\right\|^{2}
$$

For each value of $\boldsymbol{z}, G(\boldsymbol{z})$ can assume one of $N_{1} \cdot N_{2}$ values, one for each index pair to which $\boldsymbol{z}$ could be mapped. To minimize the integral it suffices to map each value of $\boldsymbol{z}$ to the index pair $(i, j)$ for which $G(\boldsymbol{z})$ is minimized. In other words,

$$
\begin{aligned}
A_{i j}=\{ & \boldsymbol{z}: \frac{p}{k}\left(k_{1}\left\|\boldsymbol{z}-\boldsymbol{z}_{i j}\right\|^{2}+k_{2} \sum_{\hat{j}=1}^{N_{2}}\left\|\boldsymbol{z}-\boldsymbol{z}_{i \hat{j}}\right\|^{2}\right)+\frac{(1-p) k_{3}}{k}\left\|\boldsymbol{z}-\boldsymbol{z}_{i}\right\|^{2} \\
& \left.\leq \frac{p}{k}\left(k_{1}\left\|\boldsymbol{z}-\boldsymbol{z}_{i^{\prime} j^{\prime}}\right\|^{2}+k_{2} \sum_{\hat{j}=1}^{N_{2}}\left\|\boldsymbol{z}-\boldsymbol{z}_{i^{\prime} \hat{j}}\right\|^{2}\right)+\frac{(1-p) k_{3}}{k}\left\|\boldsymbol{z}-\boldsymbol{z}_{i^{\prime}}\right\|^{2}, \forall\left(i^{\prime}, j^{\prime}\right) \neq(i, j)\right\} .
\end{aligned}
$$


Note that knowing $A_{i j}$ allows construction of all other partitions of the encoder using the tree structure of the MRVQ. Expanding the above equation and letting $\alpha_{i j}=p k_{1} \boldsymbol{z}_{i j}+$ $p k_{2} \sum_{j=1}^{N_{2}} \boldsymbol{z}_{i j}+(1-p) k_{3} \boldsymbol{z}_{i}, \beta_{i j}=p k_{1} \boldsymbol{z}_{i j}^{2}+p k_{2} \sum_{j=1}^{N_{2}} \boldsymbol{z}_{i j}^{2}+(1-p) k_{3} \boldsymbol{z}_{i}^{2}$. Through further simplification, we have:

$A_{i j}=\left\{\boldsymbol{z}: 2 \alpha_{i j} \cdot(\boldsymbol{z})^{\prime}-\beta_{i j} \geq 2 \alpha_{i^{\prime} j^{\prime}} \cdot(\boldsymbol{z})^{\prime}-\beta_{i^{\prime} j^{\prime}}, \forall\left(i^{\prime}, j^{\prime}\right) \neq(i, j)\right\}, \quad i=1, \cdots, N_{1}, j=1, \cdots, N_{2}$

This completes the proof of Theorem 2 .

Remark 6. The three equations (3.2), (3.3), and (3.4) in Theorem 2 reflect the complexity of designing optimal MRVQ for broadcast channels. From (3.2), (3.3), and (3.4), it follows that channel noise through different types of channel error probabilities now impacts on the update of both the quantization partition and codeword vectors at both resolution layers. On one hand, because of the impact of multiple layers in quantization, the conventional nearest neighbor encoder in single-resolution case is also not valid any more. This is in contrast with the design of optimal single resolution quantizers for noisy channels with RIA considered in [33], [34], where the update of the quantization partition is still the nearest neighbor decision rule independent of the channel. On the other hand, in comparison with the design of optimal MRVQ without reference to the channel [4], [37], the update of codeword vectors no longer follows the traditional centroid rule, in addition to the impact of channel noise on the update of the quantization partition. Note that all of these conditions target a generally good quantizer with a random index assignment rather than a given index assignment.

Remark 7. An efficient algorithm for finding the cell boundaries of such a partition in scalar case is given by Vaishampayan [37]. (3.4) gives the optimal partition for codebook which implies a tree structure. It can be shown [37] that cells of this partition consist of intervals and that if $x \in A_{i j}, y \in A_{s t}$ and $N_{2} *(i-1)+j<N_{2} *(s-1)+t$ then $x<y$. Thus for the scalar case, the interval for each $A_{i j}$ should be continuous instead of separate located.

Let $h_{i j}(x)=2 \alpha_{i j} x-\beta_{i j}$. Let $\mu(x)=\max _{i j} h_{i j}(x)$. Note that $\mu(x)$ is convex, piecewise affine a function of $x$. Hence, if $h_{i j}(x)$ coincides with $\mu(x)$, it does so over an interval of $x$ values. Note that it is possible that for some $(i, j), h_{i j}(x)$ never coincides with $\mu(x)$ for any $x$. Such index pairs are never assigned any source sample and hence are never transmitted. we conclude that $A_{i j}$ is either empty or is an interval and thus the nonempty $A_{i j}$ 's can be characterized by their endpoints.

A typical set of $h_{i j}{ }^{\prime}$ s is illustrated in Figure 3.1, where lines correspond to, in increasing order of slope, $(i, j)=(1,1),(1,2),(2,1),(2,2),(3,1),(3,2)$. It is not hard to see that index pair (1,2) and (3,1) are not transmitted.

In order to determine the endpoints of the $A_{i j}$ 's(thresholds of the central partition) we begin by identifying some of the untransmitted index pairs in the given set of index pairs $\boldsymbol{C}$. Let $\boldsymbol{B}$ be the remaining set of index pairs and let $(i, j) \in \boldsymbol{B}$ if 


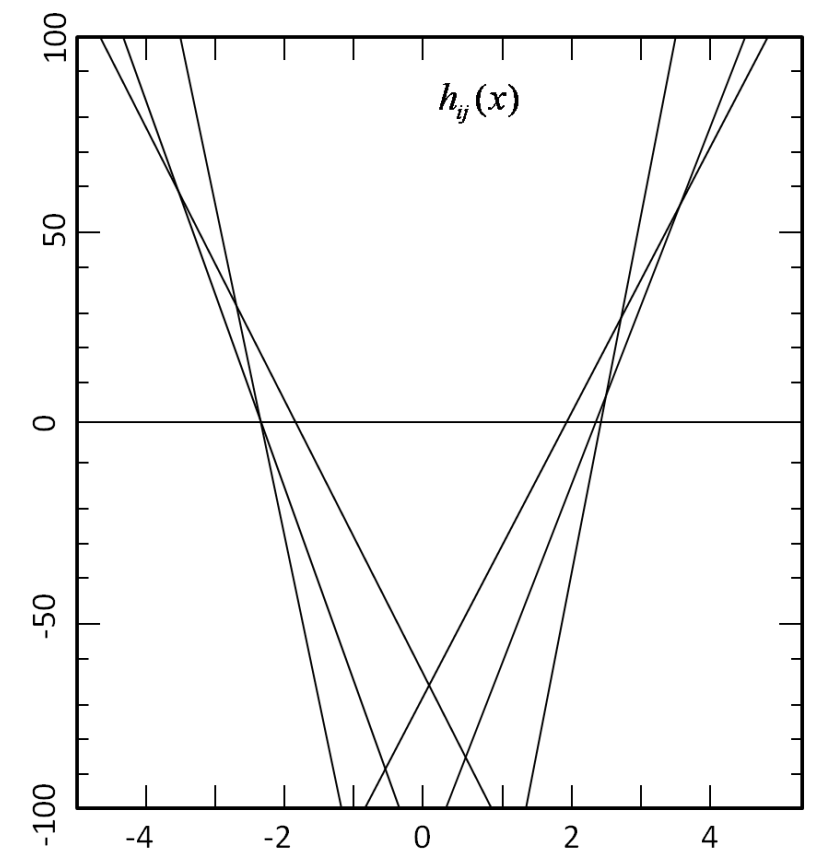

Figure 3.1: A typical set of $h_{i j}, i=1,2,3, j=1,2$

1) for all $\left(i^{\prime}, j^{\prime}\right) \in \boldsymbol{C}-(i, j), \alpha_{i j} \neq \alpha_{i^{\prime} j^{\prime}}$;

2) for all $\left(i^{\prime}, j^{\prime}\right) \in \boldsymbol{C}-(i, j), \alpha_{i j}=\alpha_{i^{\prime} j^{\prime}}, \beta_{i j}<\beta_{i^{\prime} j^{\prime}}$;

3) for all $\left(i^{\prime}, j^{\prime}\right) \in \boldsymbol{C}-(i, j), \alpha_{i j}=\alpha_{i^{\prime} j^{\prime}}, \beta_{i j}=\beta_{i^{\prime} j^{\prime}}, N_{2}(i-1)+j<N_{2}\left(i^{\prime}-1\right)+j^{\prime}$;

Define

$$
\begin{gathered}
I_{i j}^{L}=\left\{\left(i^{\prime}, j^{\prime}\right) \in \boldsymbol{B}: \alpha_{i^{\prime} j^{\prime}}<\alpha_{i j}\right\}, \\
I_{i j}^{U}=\left\{\left(i^{\prime}, j^{\prime}\right) \in \boldsymbol{B}: \alpha_{i^{\prime} j^{\prime}}>\alpha_{i j}\right\} .
\end{gathered}
$$

Let $t_{i j}^{L}$ and $t_{i j}^{U}$ be lower and upper endpoints of $A_{i j}$. Then,

$$
t_{i j}^{L}= \begin{cases}-\infty, & \text { if } I_{i j}^{L}=\emptyset, \\ \max _{\left(i^{\prime}, j^{\prime}\right) \in I_{i j}^{L}} \frac{\beta_{i j}-\beta_{i^{\prime} j^{\prime}}}{2\left(\alpha_{i j}-\alpha_{i^{\prime} j^{\prime}}\right)}, & \text { otherwise. }\end{cases}
$$

and

$$
t_{i j}^{U}= \begin{cases}\infty, & \text { if } I_{i j}^{U}=\emptyset \\ \min _{\left(i^{\prime}, j^{\prime}\right) \in I_{i j}^{U}} \frac{\beta_{i j}-\beta_{i^{\prime} j^{\prime}}}{2\left(\alpha_{i j}-\alpha_{i^{\prime} j^{\prime}}\right)}, & \text { otherwise. }\end{cases}
$$


From (3.4), it follows that

$$
A_{i j}= \begin{cases}\emptyset, & \text { if } t_{i j}^{L} \geq t_{i j}^{U}, \\ \left(t_{i j}^{L}, t_{i j}^{U}\right), & \text { if } I_{i j}^{L}=\emptyset, \\ {\left[t_{i j}^{L}, t_{i j}^{U}\right),} & \text { otherwise. }\end{cases}
$$

\subsection{Greedy Iterative Design Algorithm}

Theorem 2 suggests a gradient descent iterative algorithm for jointly designing MRVQ with channel conditions. We begin with the description of the greedy iterative algorithm for MRVQ on a broadcast channel.

The greedy iterative algorithm: In this algorithm, we update the partition $\mathfrak{A}=\left\{A_{i j}, 1 \leq\right.$ $\left.i \leq N_{1}, 1 \leq j \leq N_{2}\right\}$ and the codebook $\mathfrak{Z}_{1}=\left\{z_{i}, i=1, \cdots, N_{1}\right\}, \mathfrak{Z}_{2}=\left\{z_{i j}, i=\right.$ $\left.1, \cdots, N_{1}, j=1, \cdots, N_{2}\right\}$ of a MRVQ alternatively and iteratively according to the two necessary conditions in Theorem 2 as follows:

Step 1 Initialization: Set $\mathrm{t}=1$ and select initial partition $\left\{A_{i j}^{(1)}, 1 \leq i \leq N_{1}, 1 \leq j \leq N_{2}\right\}$ and code vectors $\left\{z_{i}^{(1)}, i=1, \cdots, N_{1},\right\},\left\{z_{i j}^{(1)}, i=1, \cdots, N_{1}, j=1, \cdots, N_{2}\right\}$. Compute $\bar{D}^{(1)}$ accordingly.

Step 2 Given $\left\{A_{i j}^{(t)}, 1 \leq i \leq N_{1}, 1 \leq j \leq N_{2}\right\}$, update the codeword vectors at both resolution layers as follows

$$
\begin{gathered}
z_{i}^{(t+1)}=\frac{\int_{A_{i}^{(t)}} z f(z) \mathrm{d} z}{\frac{p_{b}}{N_{1}-1-N_{1} p_{b}}+p\left(A_{i}^{(t)}\right)}, \quad i=1, \cdots, N_{1} \\
z_{i j}^{(t+1)}=\frac{k_{1} \int_{A_{i j}^{(t)}} z f(z) \mathrm{d} z+k_{2} \int_{A_{i}^{(t)}} z f(z) \mathrm{d} z}{k_{1} p\left(A_{i j}^{(t)}\right)+\frac{p_{e 1}}{N_{2}\left(N_{1}-1\right)}+k_{2} p\left(A_{i}^{(t)}\right)}, \quad i=1, \cdots, N_{1}, j=1, \cdots, N_{2}
\end{gathered}
$$

Step 3 Given $\left\{z_{i}^{(t+1)}, i=1, \cdots, N_{1}\right\},\left\{z_{i j}^{(t+1)}, i=1, \cdots, N_{1}, j=1, \cdots, N_{2}\right\}$, update the quantization partition $\left\{A_{i j}, 1 \leq i \leq N_{1}, 1 \leq j \leq N_{2}\right\}$ as follows

$$
\begin{gathered}
A_{i j}^{(t+1)}=\left\{z: 2 \alpha_{i j}^{(t+1)} \cdot(z)^{\prime}-\beta_{i j}^{(t+1)} \geq 2 \alpha_{i^{\prime} j^{\prime}}^{(t+1)} \cdot(z)^{\prime}-\beta_{i^{\prime} j^{\prime}}^{(t+1)}, \forall\left(i^{\prime}, j^{\prime}\right) \neq(i, j)\right\}, \\
i=1, \cdots, N_{1}, j=1, \cdots, N_{2} \\
A_{i}^{(t+1)}=\cup_{j} A_{i j}^{(t+1)}, i=1, \cdots, N_{1} .
\end{gathered}
$$




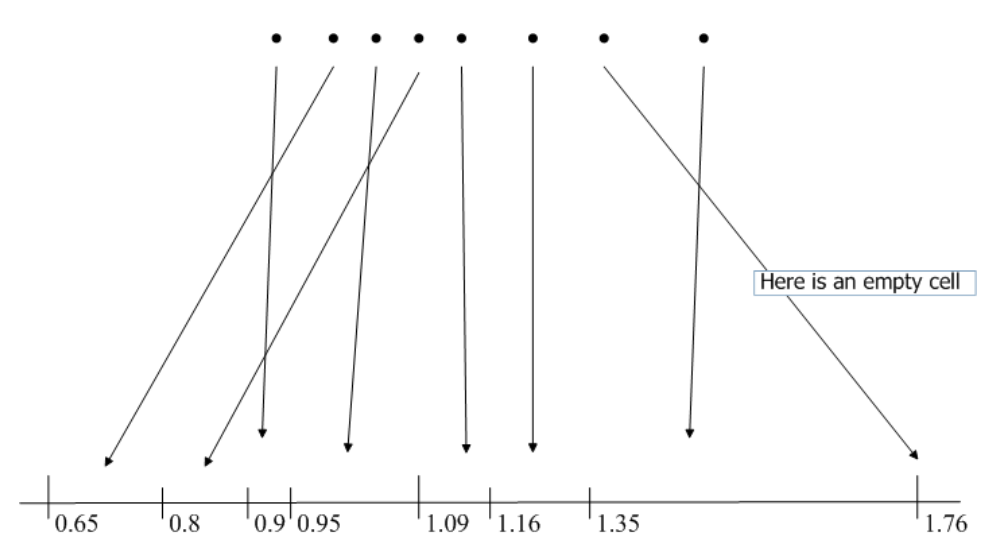

Figure 3.2: Illustration of $z_{i j} \notin A_{i j}$ and empty cells, which causes divergence for MRVQ design by alternating (3.12), (3.13), 3.14).

where,

$$
\begin{aligned}
& \alpha_{i j}^{(t+1)}=p k_{1} z_{i j}^{(t+1)}+p k_{2} \sum_{j=1}^{N_{2}} z_{i j}^{(t+1)}+(1-p) k_{3} z_{i}^{(t+1)} \\
& \beta_{i j}^{(t+1)}=p k_{1}\left\|z_{i j}^{(t+1)}\right\|^{2}+p k_{2} \sum_{j=1}^{N_{2}}\left\|z_{i j}^{(t+1)}\right\|^{2}+(1-p) k_{3}\left\|z_{i}^{(t+1)}\right\|^{2} .
\end{aligned}
$$

Step 4 If $\left|\bar{D}^{(t+1)}-\bar{D}^{(t)}\right|$ is less than some predefined threshold then $\mathrm{t}=\mathrm{t}+1$, go to Step 2; if not stop.

Start with an initial multiresolution quantizer. Repeating the step 2 and step 3, one then gets an iterative algorithm for designing optimal MRVQ for a broadcast channel through channel error probabilities. At each step in each iteration, the objective function cannot increase. Since the function is bounded below by 0 and each step produces a global minimum of $\bar{D}$ relative to the fixed components, the sequence of $\bar{D}^{(t)}$ is guaranteed to converge to a local optimum. Note that the proposed algorithm converges in the sense that the sequences of $\bar{D}^{(t)}$ are non-increasing as $t \rightarrow \infty$. In the next section, we will demonstrate the effectiveness of the proposed algorithm by presenting some experiment results.

Remark 8. Although the above iterative procedure looks similar to the Lloyd-Max algorithm [38] [39], two major differences lie in this algorithm. Firstly, it forces $z_{i}$ to move towards the origin, i.e., the mean of the source and forces $z_{i j}$ to move towards the weighed centroid of $A_{i}$. (Here we omit the superscripts $t$ and $t+1$ for convenience.) As a result, during the iterative procedure, the updated $z_{i}$ or $z_{i j}$ at each step given $A_{i}$ and $A_{i j}$ may not fall into $A_{i}$ or $A_{i j}$, 
especially when the error probabilities are large. Secondly, the partitions are not midpoints of two representative points. Moreover it is possible that some intervals do not exist. Thus if only applying these steps (3.12) (3.13) (3.14), an unexpected case occurs that some cells of the partition may be empty. Actually this phenomenon is reasonable as discussed later. However if one wants to eliminate empty cells, it necessitates some modifications in the algorithm. For the design of successively refinable scalar quantizers [37], if an empty cell occurs in some steps, the parent's cell of an empty cell will be uniformly partitioned. While in order to make sequences of $\left\{A_{i j}\right\}$ and $\left\{z_{i j}\right\}$ also converge, now we oblige an empty cell to be a non-empty cell of length $\delta$ that is arbitrarily small, by compressing its neighbor. The above discussions are further illustrated in Figure 3.2 for partitions and quantization codebook obtained at some step of the above iterative procedure when it is applied to a 2-resolution quantizer with $4 \times 4$ levels for a Gaussian source with zero mean and unit variance over a broadcast channel. In Figure 3.2, the horizontal line with numbers shows the partition(longer segments for base layer, shorter ones for enhancement layer), while the dots represent the updated reproduction points at enhancement layer given the partitions. As shown by the arrows, there are three partition sets, for which the respective updated quantization outputs are outside each respective set, i.e., the first, second, and the last. Therefore, the subsequent alternating steps will make $\left\{A_{i j}, 1 \leq i \leq N_{1}, 1 \leq j \leq N_{2}\right\}$ and $\left\{z_{i j}, 1 \leq i \leq N_{1}, 1 \leq j \leq N_{2}\right\}$ appear in random order along the horizontal line. This in turn will make it difficult to analyze the convergence of the corresponding sequence of quantizers.

\subsection{Simulations}

Simulations have been conducted to compare the performance of quantizers designed by the proposed algorithms with that of quantizers designed by the conventional MRVQ algorithm. Given a quantizer and the error probabilities $p_{e 1}, p_{e 2}$ and $p_{b}$, the weighted EED for random index assignment is computed using (2.11). All experiments have been carried out for one-dimensional case, and we will demonstrate the relationships between the weighted distortion and each error probability above when fixing the other variables.

Consider a one-dimensional Gaussian source with zero mean and unit variance. Figure 3.3. Figure 3.4 and Figure 3.5 show the gain by PSNR, which is defined as PSNR = $10 \log _{10}\left(\sigma^{2} / \bar{D}\right)$, where $\sigma^{2}$ is the signal variance and $\bar{D}$ is the weighted distortion. For a given quantizer, $\bar{D}$ is computed using 2.11). As shown in the simulation results, the weighted distortion increases as either $p_{e 1}$ or $p_{b}$ grows up, however it indicates that the weighed distortion keeps constant while $p_{e 2}$ goes up. It seems that the effect of $p_{e 2}$ on the weighted distortion is much less then $p_{e 1}$ and $p_{b}$. It sounds reasonable, since if the transmission error just occurs at the enhancement layer, the reproduction point must lie 
not far from the transmitted point, in the same region as the transmitted data on the base layer. Thus, such error induces only a little distortion, relatively less than that with an error at base layer. Besides, from these figures it is shown that the gain of the proposed MRVQ design over the algorithm designed separately without reference to the channel conditions is significant. Table 3.1 indicates that the gain (in $d B$ ) of the proposed MRVQ design over the algorithm designed separately goes more and more as either $N_{1}$ or $N_{2}$ increases. As shown in the table, the number of quantization levels at base layer plays a more important role than that at enhancement layer in the distortion performance. For example, even if the total quantization levels are the same for the quantizer with $N_{1}=16$ and $N_{2}=8$ and the quantizer with $N_{1}=32$ and $N_{2}=4$, however, the latter one generates smaller distortion for both the Greedy iterative algorithm and the algorithm designed separately without reference to the channel conditions obviously and larger gain between these two algorithms.

Table 3.1: PSNR gain of our proposed MRVQ design over the separate MRVQ design for Gaussian source with zero mean and unit variance given $p_{e 1}=0.01, p_{e 2}=0.005$ and $p_{b}=0.02$.

\begin{tabular}{l|l|l|l|l|l|l}
\hline & $N_{1}=4$ & $N_{1}=8$ & $N_{1}=16$ & $N_{1}=16$ & $N_{1}=32$ & $N_{1}=32$ \\
& $N_{2}=2$ & $N_{2}=2$ & $N_{2}=2$ & $N_{2}=8$ & $N_{2}=4$ & $N_{2}=8$ \\
\hline Proposed MRVQ design & 9.1937 & 11.7653 & 13.3724 & 13.8934 & 14.8413 & 14.9894 \\
\hline Separate MRVQ design & 9.1009 & 11.4691 & 12.3582 & 12.4109 & 12.5104 & 12.5181 \\
\hline Our PSNR gain & 0.0928 & 0.2962 & 1.0142 & 1.4825 & 2.3309 & 2.4713 \\
\hline
\end{tabular}

\section{4 "Empty Cells" Phenomenon}

It is shown in my simulation results that there may exist several empty cells in the quantizer encoder, that is, some partitions are empty in that some codevectors emerge. As a similar phenomenon occurred in single resolution case, it was observed experimentally in 32 . that for channel optimized quantizers, as the transmission rate increased, the number of quantization levels would at some point not increase any more. Instead the additional rate available was better used in channel coding. Adding more quantization levels would in fact have a detrimental effect in such cases. This observation is supported by Corollary 3 in 32], which shows that the mean square error (MSE) of quantization can actually increase in the presence of channel noise as the number of levels is increased. The average distortion $D(N)$ is strictly increasing as a function $N$ for $N>N_{\text {opt }}$, that is, increasing $N$ beyond the optimal number of levels only reduces the performance. 

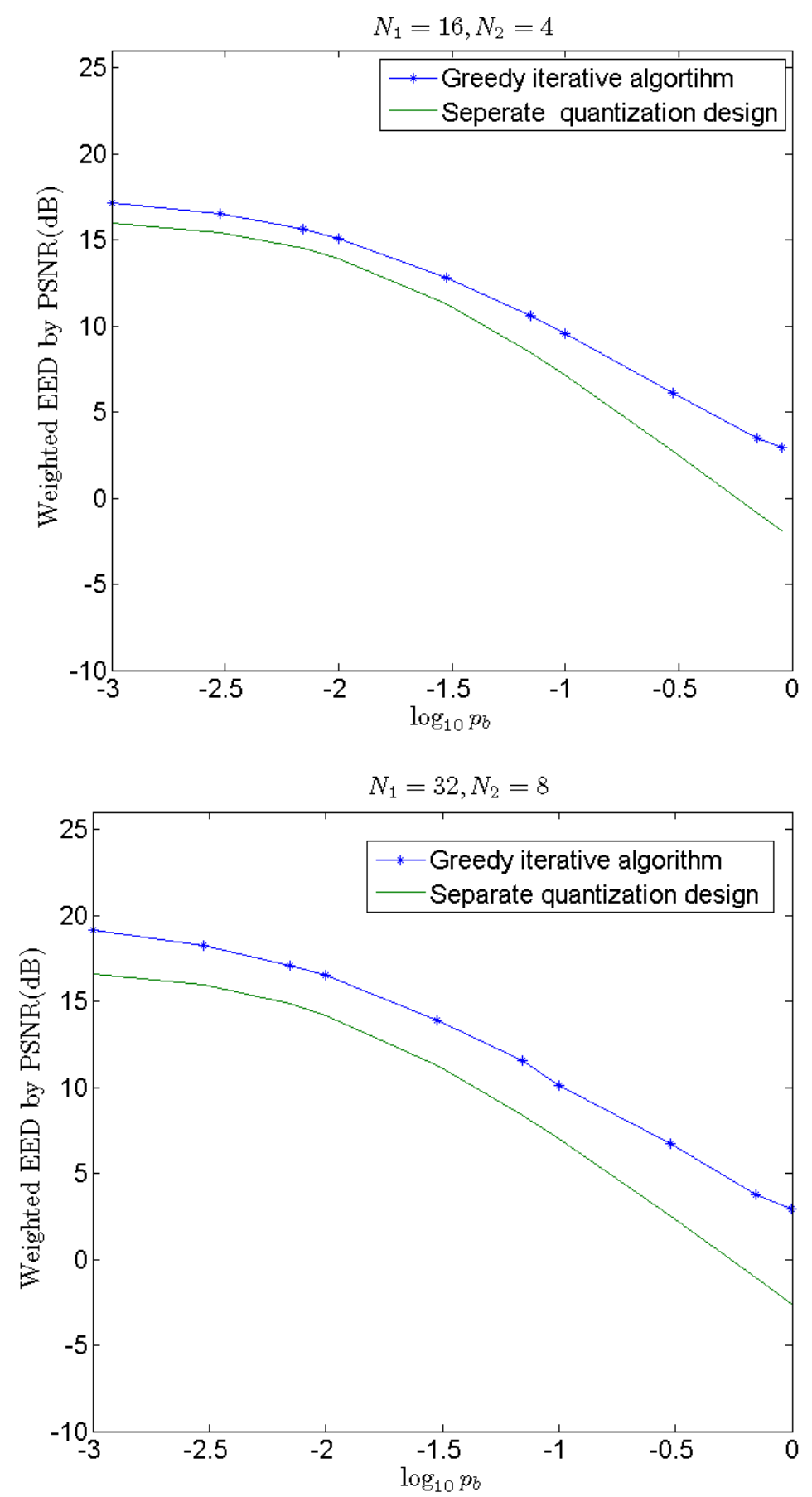

Figure 3.3: PSNR gain of channel optimized quantization over quantization designed separately from channels corresponding to various $p_{b}$ for a Gaussian source while fixing $p=0.5$, $p_{e 1}=0.01$ and $p_{e 2}=0.005$ 

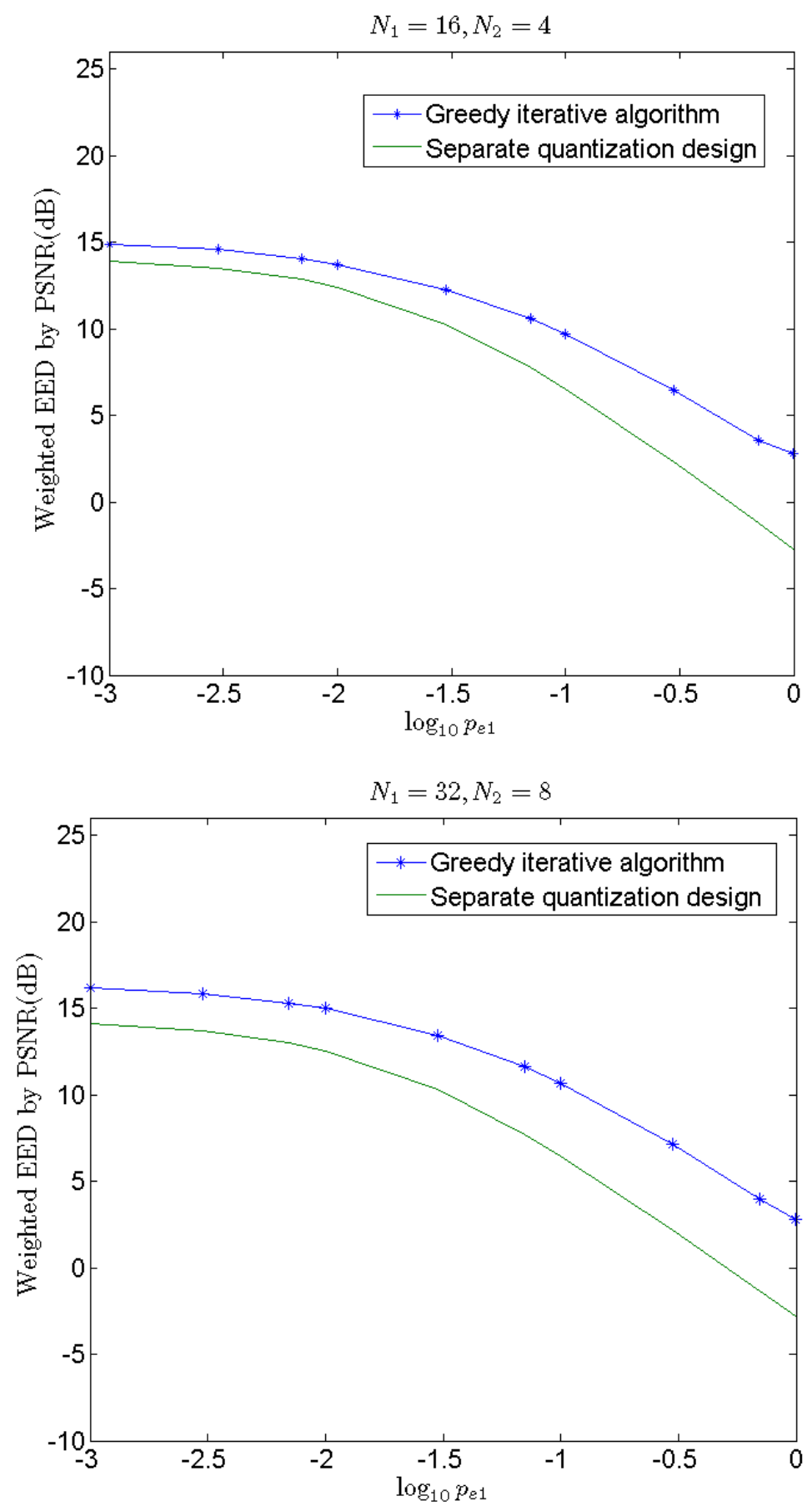

Figure 3.4: PSNR gain of channel optimized quantization over quantization designed separately from channels corresponding to various $p_{e 1}$ for a Gaussian source while fixing $p=0.5$, $p_{b}=0.02$ and $p_{e 2}=0.005$ 

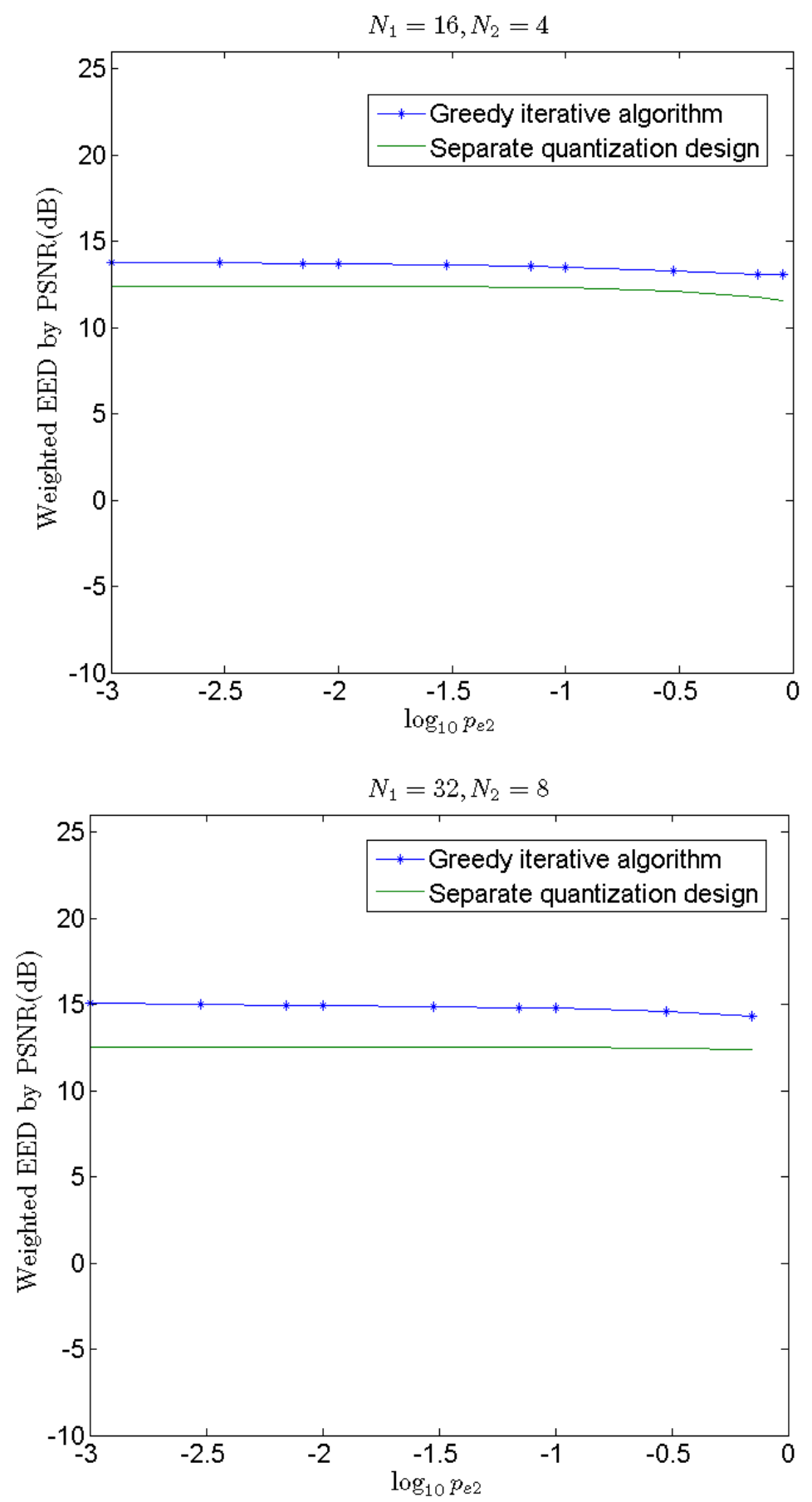

Figure 3.5: PSNR gain of channel optimized quantization over quantization designed separately from channels corresponding to various $p_{e 2}$ for a Gaussian source while fixing $p=0.5$, $p_{b}=0.02$ and $p_{e 1}=0.01$ 
Consider a vector quantizer source coding system with a channel coder that transmits data across channel. Let $R_{s}$ denote the source rate, i.e., the number of bits per input vector component used for vector quantization. Let $\mathrm{R}$ denote the channel usage rate, i.e., the number of binary channel uses per input vector component. Let $R_{c}=R_{s} / R$ be the channel code rate, i.e., the fraction of transmitted bits that are used as information bits for source coding. The vector quantizer has a codebook of size $2^{k R_{s}}$ and the overall transmission rate of the system is $\mathrm{R}$, where in general $R_{s} \leq R$. The difference $R-R_{s}$, is the number of redundancy bits per vector component used for error correction coding. If a redundancy free channel code is used then $R_{s}=R$, such as when the channel is noiseless.

If the overall transmission rate $\mathrm{R}$ of the system is fixed and we wish to minimize $\bar{D}$, the choice of source rate $R_{s}$ or equivalently channel code rate $R_{c}$ trades off between source and channel coding. If one transmits at a rate $R_{c}$ very close to capacity $\mathrm{C}$, then the number of information bits $R_{s}$, will be large, and thus the quantization error $E\|X-Y\|^{2}$ will be small; however, for large $R_{c}$, the probability of an uncorrected channel error cannot be as tightly upper bounded (smaller error exponent), so that average error probabilities, will contribute more to the overall distortion $\bar{D}$. Thus, there is an important trade-off in this case between: i) designating more of the transmitted bits as information bits to reduce quantization error, and ii) devoting more of the transmitted bits toward error control coding to drive the probability of an uncorrected channel error to zero.

As it is demonstrated in my simulation results, we assume a set of average error probabilities $p_{e 1}, p_{e 2}, p_{b}$ based on a specific coding scheme with $R_{s}$ and $R_{c}$. For the channel optimized quantization design, there may exist several empty cells in the quantizer encoder. It means that we would not make use of these empty cells to encode a sequence of source variables, that is, these cells are actually invalid for compression. Besides, as the number of multiresolution quantization levels grows up, the quantity of the empty cells increases as well. An intuitive explanation for this is that as the rate increases, the resulting longer codevector indices are more exposed to damaging channel errors. Thus, over channels with smaller average probabilities, one can transmit longer indices to achieve the minimum MSE; on channels with larger probabilities, a part of source rate might act as error protection against the channel noise.

The optimal codewords $\left\{z_{i j}\right\}$ associated with a channel optimized vector quantization will change as a function of three average index crossover probabilities $p_{e 1}, p_{e 2}, p_{b}$. In particular, the codewords will tend to be closer when the probability of an average index error is high than when that same probability is low. For sufficiently high average error probabilities, the codewords will actually merge, meaning that two or more codeword indices (e.g. $(i, j)$ and $(i, j+1))$ will map to the same reproduction vector (e.g. $z_{i j}=z_{i, j+1}$ ). A source code with two or more identical codewords effectively uses some of the source coding bits for redundancy or channel error protection. In this case we say that the effective source coding rate $R_{e}$ is lower than the source coding rate $R_{s}$, since the source code applies 
some of its rate $R_{s}-R_{e}$ to the job of error protection. Therefore the total number of bits applied to channel error protection is $R-R_{e}$ : we call this the effective channel coding rate. Given a quantization with distinct codewords, we will calculate the effective source coding rate as $R_{e}=\log N / k$. For the jointly designed quantization, as $R_{s}$ increases the channel coding rate $R_{c}$ increases, and thus, the average error probabilities increase. This will eventually cause some of the source bits to be used for error protection so that, above some critical $R_{s}$ value, $R_{e}$ will drop below $R_{s}$, as we observe in the experiments. Therefore, as discussed above, the empty cell phenomenon is a natural property of channel-optimized source coding.

\subsection{Comparison With The KR Algorithm}

It may be of some interests to compare the proposed method with the multi-resolution source codebook design in [36], which assumes a fixed index assignment. Apparently, the KR algorithm requires the complete knowledge of the channel, i.e., all transitional probabilities, which is usually unavailable in practice. On the other hand, the average channel information, on which the proposed method is based, is relatively easy to obtain.

Since the KR algorithm needs all transitional probabilities $p_{\pi_{t}}\left(z_{\hat{i} \hat{j}} \mid z_{i j}\right)$, the first step in this experiment is to assume these transitional probabilities. For example, to design a 2-resolution scalar quantization with $4 \times 4$ levels, we generate randomly three pairs of transitional probability matrices, $\left(\mathbf{P}_{\mathbf{i e}}, \mathbf{P}_{\mathbf{i b}}\right), i=1,2,3$. These pairs $\left(\mathbf{P}_{\mathbf{i e}}, \mathbf{P}_{\mathbf{i b}}\right)$ 's correspond to three channel conditions with slight noise, mediate noise and severe noise respectively, where each $\mathbf{P}_{\mathbf{i e}}$ is a $16 \times 16$ matrix and each $\mathbf{P}_{\mathbf{i b}}$ is a $4 \times 4$ matrix. Due to the limit of space and also for brevity, I do not list these three pairs of matrices.

Given these transitional probabilities, we then apply the KR algorithm to a Gaussian source to get the respective quantizer by using the natural index assignment mapping, and the actual EED of which is finally calculated according to $p D_{e}^{\pi_{t}}+(1-p) D_{b}^{\pi_{t}}$. To apply our proposed algorithm to the Gaussian source, we just need the average symbol error probabilities, which correspond to these transitional probabilities. The actual EED of the quantizers designed is calculated according to (2.1) and (2.2). In Table 3.2 shown are the actual EEDs of the quantizers designed by our proposed algorithm, the KR algorithm, and separate quantization design without reference to the channel conditions. As expected, the quantizers designed by the KR algorithm outperform sightly the quantizers designed by our proposed algorithm. However, what makes this comparison interesting is that the performance gain is marginal compared to the gain of the quantizers by our proposed algorithm over the quantizer designed by separate quantization design. In addition, this marginal performance gain is achieved with much higher design complexity and with complete knowledge of all transitional probabilities. For example, to design a 2-resolution 
quantization with $N_{1} \times N_{2}$ levels, the $\mathrm{KR}$ algorithm requires $\mathcal{O}\left(N_{1}^{2} N_{2}^{2}\right)$ arithmetic operations, while the proposed one consumes $\mathcal{O}\left(N_{1} N_{2}\right)$, so the computational complexity of the proposed method is much less than the KR algorithm.

Table 3.2: Performance comparison in terms of PSNR (dB) for MRVQ designed by our proposed algorithm, the KR algorithm, and separate MRVQ design.

\begin{tabular}{l|l|l|l}
\hline & $\left(\mathbf{P}_{\mathbf{1 e}}, \mathbf{P}_{\mathbf{1 b}}\right)$ & $\left(\mathbf{P}_{\mathbf{2 e}}, \mathbf{P}_{\mathbf{2 b}}\right)$ & $\left(\mathbf{P}_{\mathbf{3 e}}, \mathbf{P}_{\mathbf{3 b}}\right)$ \\
& s.t. $p_{b}=0.002$, & s.t. $p_{b}=0.02$, & s.t. $p_{b}=0.2$, \\
& $p_{e 1}=0.001$, & $p_{e 1}=0.01$, & $p_{e 1}=0.1$, \\
& $p_{e 2}=0.0015$ & $p_{e 2}=0.015$ & $p_{e 2}=0.15$ \\
\hline KR algorithm & 11.656 & 9.736 & 4.160 \\
\hline Proposed algorithm & 11.656 & 9.706 & 4.057 \\
\hline Separate quantization design & 11.650 & 9.530 & 2.662 \\
\hline
\end{tabular}

In practice, the complete knowledge of all transitional probabilities may be hard to get. Even if transitional probabilities are available, they will fluctuate in wireless communications. As such, it is also interesting to look at how sensitive our proposed algorithm, KR algorithm and separate quantization design are with respect to channel noise fluctuation. Table 3.3 shows the actual EEDs by PSNR in $d B$ when a quantizer designed for a set of transitional probabilities by either the KR algorithm or our proposed algorithm is applied to a less noisy channel environment with $\left(\mathbf{P}_{\mathbf{4 e}}, \mathbf{P}_{\mathbf{4 b}}\right)$ or a more noisy channel environment with $\left(\mathbf{P}_{\mathbf{5 e}}, \mathbf{P}_{\mathbf{5 b}}\right)$. For a clearer comparison, we also repeat here the results as shown in the second column of Table 3.3. It is interesting to see that the quantizer designed by our proposed algorithm actually outperforms that by the KR algorithm for both more noisy channel environment and less noisy channel environment. In other words, the quantizer designed by the KR algorithm for a specific channel deteriorated its performance to a larger extend when channel noise fluctuates, which makes it vulnerable in wireless communications, while the proposed method shows better robustness against the channel fluctuation than the KR algorithm simply by the nature of using the average channel information.

Therefore, with the above experiment and simulation results, we can reasonably conclude that the proposed algorithm is more appropriate in both wireless communications and applications where the complete knowledge of the channel is hard to obtain.

\subsection{Novel Iterative Algorithms}

As discussed above, the algorithm generalized from Lloyd-Max algorithm converges to a local optimal solution, and at this point it is hardly possible to prove the uniqueness of the 
Table 3.3: Sensitivity comparison in term of PSNR (dB) for MRVQ designed by our proposed algorithm and the KR algorithm.

\begin{tabular}{l|l|l|l}
\hline & $\left(\mathbf{P}_{\mathbf{4 e}}, \mathbf{P}_{\mathbf{4 b}}\right)$ & $\left(\mathbf{P}_{\mathbf{2 e}}, \mathbf{P}_{\mathbf{2 b}}\right)$ & $\left(\mathbf{P}_{\mathbf{5 e}}, \mathbf{P}_{\mathbf{5 b}}\right)$ \\
& s.t. $p_{b}=0.002$, & s.t. $p_{b}=0.02$, & s.t. $p_{b}=0.03$, \\
& $p_{e 1}=0.001$, & $p_{e 1}=0.01$, & $p_{e 1}=0.015$, \\
& $p_{e 2}=0.0015$ & $p_{e 2}=0.015$ & $p_{e 2}=0.0225$ \\
\hline KR algorithm & 10.101 & 9.736 & 9.274 \\
\hline Proposed algorithm & 10.186 & 9.706 & 9.292 \\
\hline
\end{tabular}

local minimal points, so we cannot guarantee whether this is the best algorithm for Multiresolution quantization on noisy channels or not. That implies that maybe there exist some other algorithms which generate different encoders and decoders to achieve better distortion performance. After lots of discussions, inspired by the idea of the scalable video coding in H.264, two novel iterative algorithms come up. One of them aims to optimize the quantizers at two layers alternatively and iteratively, that is, update the representative points and partitions at one layer given those at the other layer and vice verse; and the other satisfies the constraint that each encoding cell is convex and contains the reconstruction point. We describe these two new iterative algorithms first, then experiments are conducted to compare our proposed algorithms in different aspects.

\subsubsection{New iterative algorithm}

In this algorithm, we optimize low-resolution quantization given the high-resolution quantization and vice versa. The quantizers on each layer are updated alternatively and iteratively as follows.

The new iterative algorithm:

Step 1 Initialization: Set $\mathrm{t}=1$ and select initial partition $\left\{A_{i j}^{(1)}, 1 \leq i \leq N_{1}, 1 \leq j \leq N_{2}\right\}$ and code vectors $\left\{z_{i}^{(1)}, i=1, \cdots, N_{1}\right\},\left\{z_{i j}^{(1)}, i=1, \cdots, N_{1}, j=1, \cdots, N_{2}\right\}$. Compute $\bar{D}^{(1)}$ accordingly.

Step 2 Given $\left\{z_{i j}^{(t)}, i=1, \cdots, N_{1}, j=1, \cdots, N_{2}\right\}$, update the $\left\{z_{i}^{(t+1)}, i=1, \cdots, N_{1}\right\},\left\{A_{i}^{(t+1)}, i=\right.$ $\left.1, \cdots, N_{1}\right\}$ by performing the following steps iteratively.

1) Given $\left\{A_{i}^{(\hat{t})}, i=1, \cdots, N_{1}\right\}$, the optimal codeword vectors for minimizing $\bar{D}$ at 
base layer are computed respectively by

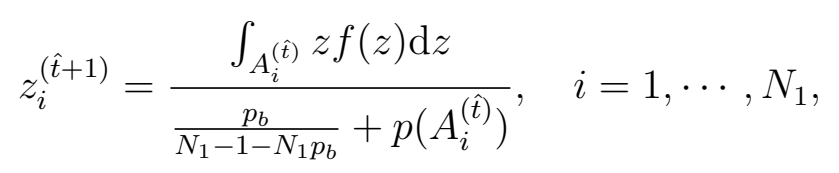

2) Given $\left\{z_{i}^{(\hat{t}+1)}, i=1, \cdots, N_{1}\right\}$, the optimal partition is achieved by

$$
\begin{gathered}
A_{i j}^{(\hat{t}+1)}=\left\{z: 2 \alpha_{i j}^{(\hat{t}+1)} \cdot(z)^{\prime}-\beta_{i j}^{(\hat{t}+1)} \geq 2 \alpha_{i^{\prime} j^{\prime}}^{(\hat{t}+1)} \cdot(z)^{\prime}-\beta_{i^{\prime} j^{\prime}}^{(\hat{t}+1)}, \forall\left(i^{\prime}, j^{\prime}\right) \neq(i, j)\right\}, \\
i=1, \cdots, N_{1}, j=1, \cdots, N_{2} \\
A_{i}^{(\hat{t}+1)}=\cup_{j} A_{i j}^{(\hat{t}+1)}, i=1, \cdots, N_{1} .
\end{gathered}
$$

Step 3 Given $\left\{z_{i}^{(t+1)}, i=1, \cdots, N_{1}\right\},\left\{A_{i}^{(t+1)}, i=1, \cdots, N_{1}\right\}$, update the $\left\{z_{i j}^{(t+1)}, i=1, \cdots, N_{1}, j=\right.$ $\left.1, \cdots, N_{2}\right\},\left\{A_{i j}^{(t+1)}, 1 \leq i \leq N_{1}, 1 \leq j \leq N_{2}\right\}$ by performing the following steps iteratively. For simplicity, write $A_{i}, z_{i}$ short for $A_{i}^{(t+1)}, z_{i}^{(t+1)}$ respectively in the following sub-steps.

1) Given $\left\{A_{i j}^{(\hat{t})}, 1 \leq i \leq N_{1}, 1 \leq j \leq N_{2}\right\}$, the optimal codeword vectors for minimizing $\bar{D}$ at enhancement layer are computed respectively by

$$
\begin{array}{r}
z_{i j}^{(\hat{t}+1)}=\frac{k_{1} \int_{A_{i j}^{(\hat{t}}} z f(z) \mathrm{d} z+k_{2} \int_{A_{i}} z f(z) \mathrm{d} z}{k_{1} p\left(A_{i j}^{(\hat{t})}\right)+\frac{p_{e 1}}{N_{2}\left(N_{1}-1\right)}+k_{2} p\left(A_{i}\right)} \\
\quad i=1, \cdots, N_{1}, j=1, \cdots, N_{2} .
\end{array}
$$

2) Given $\left\{z_{i j}^{(\hat{t}+1)}, i=1, \cdots, N_{1}, j=1, \cdots, N_{2}\right\}$, the optimal finest partition is achieved by

$$
\begin{array}{r}
A_{i j}^{(\hat{t}+1)}=\left\{z:\left|z-z_{i j}^{(\hat{t}+1)}\right|^{2} \leq\left|z-z_{i j^{\prime}}^{(\hat{t}+1)}\right|^{2}, \forall j^{\prime} \neq j\right\} \\
i=1, \cdots, N_{1}, j=1, \cdots, N_{2},
\end{array}
$$

Step 4 If $\left|\bar{D}^{(t+1)}-\bar{D}^{(t)}\right|$ is less than some predefined threshold then $\mathrm{t}=\mathrm{t}+1$, go to Step 2; if not stop.

Remark 9. According to the idea that update the regions and codebook at each layer iteratively and alternatively, it is natural to optimize the objective function by choosing the regions and codebook at one layer by fixing the quantizer at the other layer. When fixing the quantizer at base layer and updating the quantizer at enhancement layer, this process 
is sensible. We only need to proceed the one dimensional quantization algorithm to update $\left\{z_{i j}, i=1, \cdots, N_{1}, j=1, \cdots, N_{2}\right\}$, and $\left\{A_{i j}, i=1, \cdots, N_{1}, j=1, \cdots, N_{2}\right\}$ alternatively for a large number of iterations. Note that in this process the update of the $A_{i j}$ 's follows the traditional nearest neighborhood rule, so the update function is simplified to (3.18).

However, given $\left\{z_{i j}, i=1, \cdots, N_{1}, j=1, \cdots, N_{2}\right\},\left\{A_{i j}, i=1, \cdots, N_{1}, j=1, \cdots, N_{2}\right\}$, to update $\left\{z_{i}, i=1, \cdots, N_{1}\right\},\left\{A_{i}, i=1, \cdots, N_{1}\right\}$ induces some problems. Because of the constraint that $A_{i}=\cup_{j} A_{i j}, i=1, \cdots, N_{1}$, if $\left\{A_{i j}, i=1, \cdots, N_{1}, j=1, \cdots, N_{2}\right\}$ is fixed, then $\left\{A_{i}, i=1, \cdots, N_{1}\right\}$ cannot be recalculated. Therefore, in this algorithm, a small modification is made, that is, at Step 2 we only fix $\left\{z_{i j}, i=1, \cdots, N_{1}, j=1, \cdots, N_{2}\right\}$, and relax $\left\{A_{i j}, i=1, \cdots, N_{1}, j=1, \cdots, N_{2}\right\}$. Actually, we update the partitions at base layer, at the same time the partitions at enhancement layer are also modified.

Simulations have been conducted to compare the performance of quantizers designed by new iterative algorithms with that of quantizers designed by new iterative algorithm. Given a quantizer and average error probabilities $p_{e 1}, p_{e 2}$ and $p_{b}$, the weighted EED for random index assignment is computed using (2.11). All experiments have been carried out for one-dimensional case, and we will demonstrate the relationships between the weighted distortion and each error probability above when fixing the other variables.

Consider a one-dimensional Gaussian source with zero mean and unit variance. Figure 3.6 and Figure 3.7 show the performance of greedy iterative algorithm and new iterative algorithm by PSNR. As shown in the simulation results, the weighted distortion of new iterative algorithm increases as either $p_{e 1}$ or $p_{b}$ grows up.

It is shown that these two iterative algorithms obtain almost the same performance. After examining the data, new iterative algorithm is actually better than the greedy one so slightly that the difference in the weighted distortion can hardly be distinguished. Moreover, by checking the quantization partitions and reconstruction points carefully, we find that these results are completely different from those of greedy iterative algorithm. In addition, the phenomenon of empty cells still exists in the final results generated by new iterative algorithm, but the number of empty cells drops down sharply in comparison with greedy iterative algorithm. It is interesting to observe that each algorithm converges to a local optimum but different from each other. This indicated that there are several local minimal points for the objective function in terms of $A_{i j}$ and $z_{i j}$, so it is not correct to assert either algorithm converges to a global optimum without any proof.

As discussed previously, the rate for empty cells are used to fight against noises in the transmission. However, though it is a reasonable explanation, in some scenarios a regular vector quantizer is indeed required [a quantizer is regular if each encoding cell is convex and contains the reconstruction point]. Thus, the empty cells seem to cause problems in the cases where regular quantizers are preferred. To overcome the above problem, we shall 

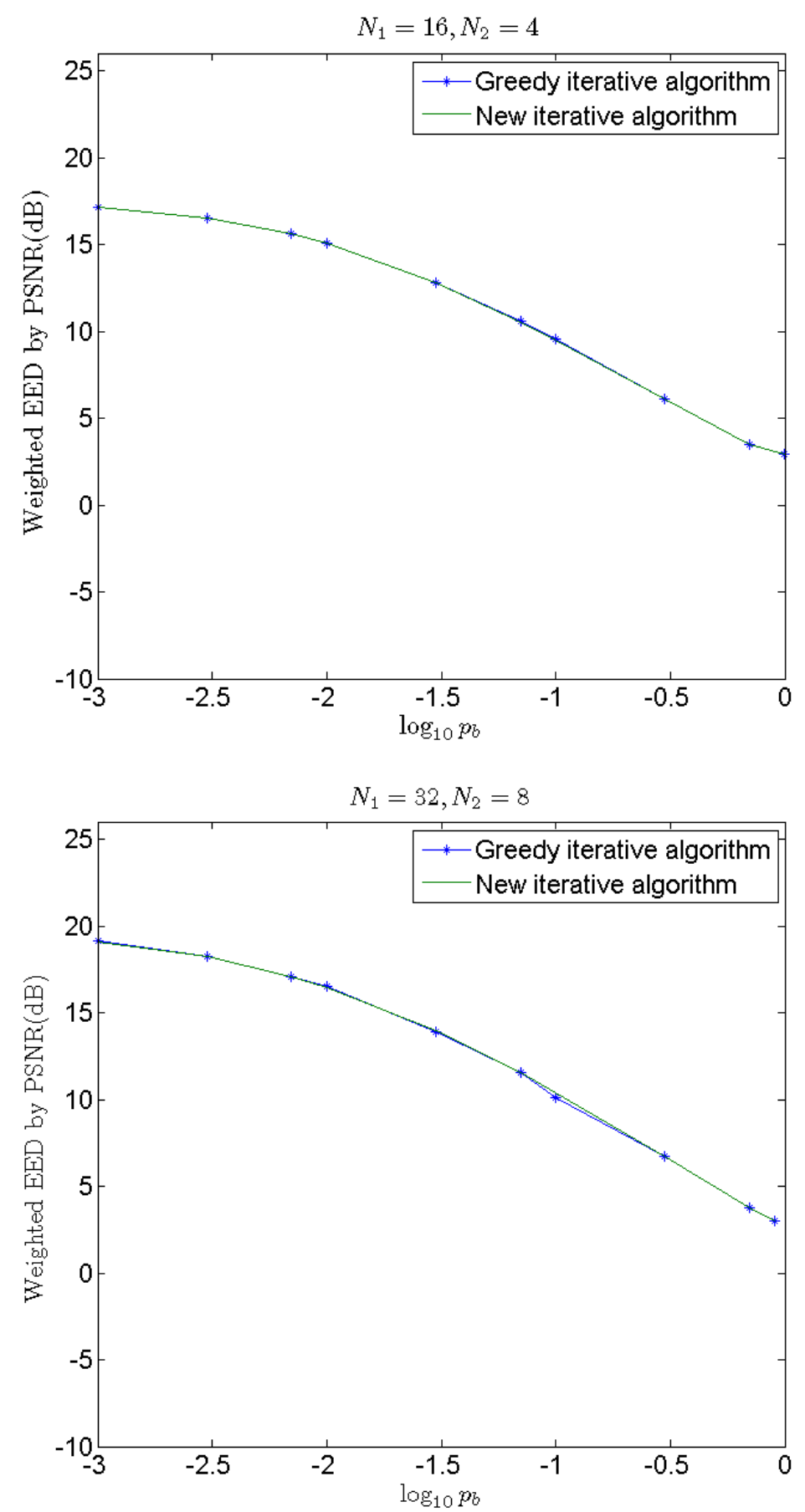

Figure 3.6: Comparison between greedy iterative algorithm and new iterative algorithm corresponding to various $p_{b}$ for a Gaussian source while fixing $p=0.5, p_{e 1}=0.01$ and $p_{e 2}=0.005$ 

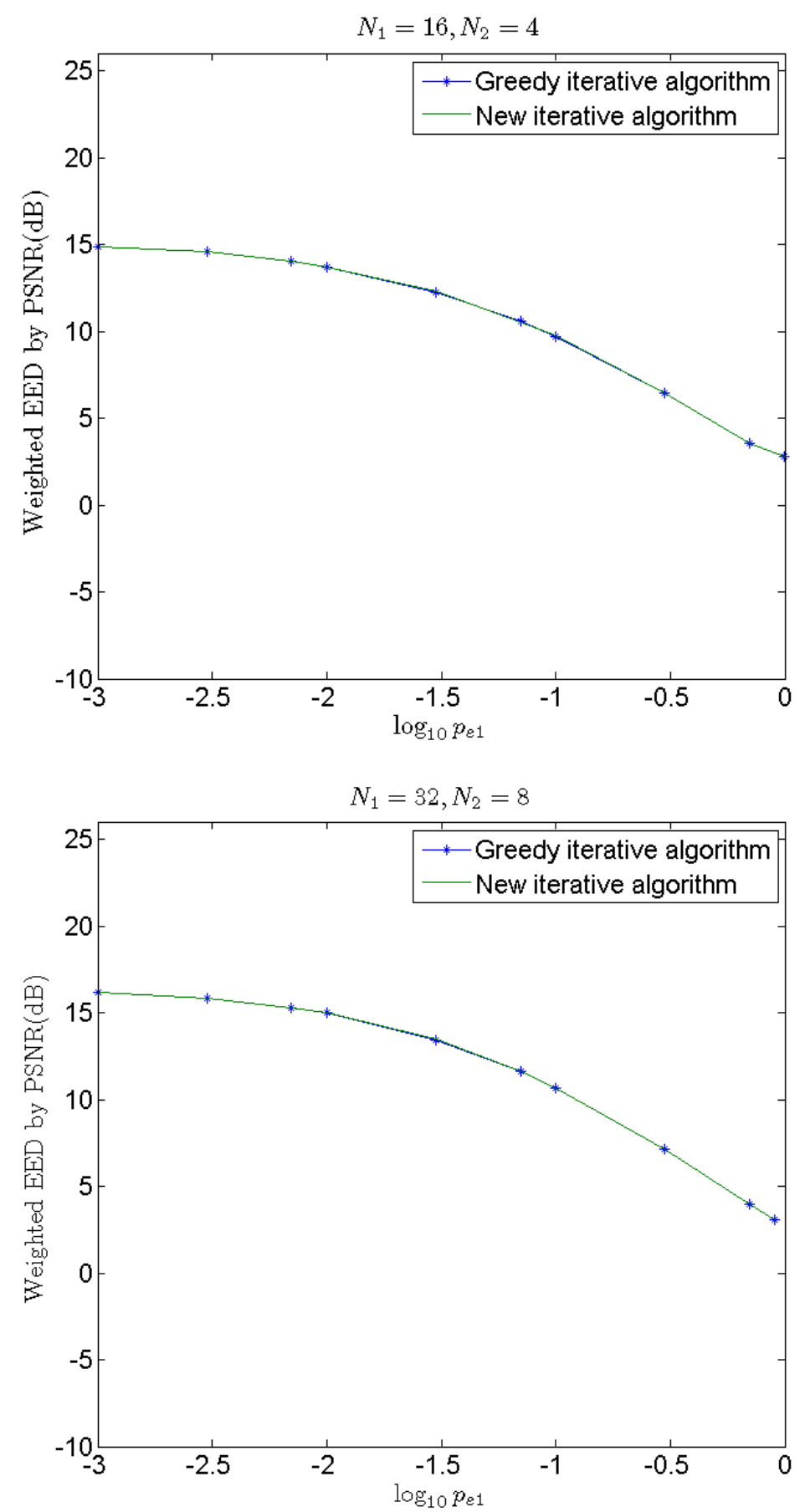

Figure 3.7: Comparison between greedy iterative algorithm and new iterative algorithm corresponding to various $p_{e 1}$ for a Gaussian source while fixing $p=0.5, p_{b}=0.02$ and $p_{e 2}=0.005$ 
force each updated $z_{i j}$ to be within the given $A_{i j}$ and each updated $z_{i}$ to be within the given $A_{i}$, yielding our controlled iterative algorithm to design a regular quantizer.

\subsubsection{Controlled iterative algorithm}

This algorithm applies under the constraint that each encoding cell is convex and contains the reconstruction point. In this algorithm, we force each updated $z_{i j}$ to be within the given $A_{i j}$ and each updated $z_{i}$ to be within the given $A_{i}$, and update the quantizers on each layer alternatively and iteratively as follows.

The Controlled Iterative Algorithm:

Step 1 Initialization: Set $\mathrm{t}=1$ and select initial partition $\left\{A_{i j}^{(1)}, 1 \leq i \leq N_{1}, 1 \leq j \leq N_{2}\right\}$ and code vectors $\left\{z_{i}^{(1)}, i=1, \cdots, N_{1}\right\},\left\{z_{i j}^{(1)}, i=1, \cdots, N_{1}, j=1, \cdots, N_{2}\right\}$. Compute $\bar{D}^{(1)}$ accordingly.

Step 2 Given $\left\{z_{i j}^{(t)}, i=1, \cdots, N_{1}, j=1, \cdots, N_{2}\right\}$, update the $\left\{z_{i}^{(t+1)}, i=1, \cdots, N_{1}\right\},\left\{A_{i}^{(t+1)}, i=\right.$ $\left.1, \cdots, N_{1}\right\}$ by performing the following steps iteratively.

1) Given $\left\{A_{i}^{(\hat{t})}, i=1, \cdots, N_{1}\right\}$, the optimal codeword vectors for minimizing $\bar{D}$ at base layer are computed respectively by

$$
z_{i}^{(\hat{t}+1)}=\arg \min _{\hat{z} \in A_{i}^{(\hat{t})}}\left(1-\frac{N_{1} p_{b}}{N_{1}-1}\right) \int_{z \in A_{i}^{(\hat{t})}}\|z-\hat{z}\|^{2} f(z) \mathrm{d} z+\frac{p_{b}}{N_{1}-1}\|\hat{z}\|^{2}, \quad i=1, \cdots, N_{1},
$$

2) Given $\left\{z_{i}^{(\hat{t}+1)}, i=1, \cdots, N_{1}\right\}$, the optimal partition is achieved by

$$
\begin{gathered}
A_{i j}^{(\hat{t}+1)}=\left\{z: 2 \alpha_{i j}^{(\hat{t}+1)} \cdot(z)^{\prime}-\beta_{i j}^{(\hat{t}+1)} \geq 2 \alpha_{i^{\prime} j^{\prime}}^{(\hat{t}+1)} \cdot(z)^{\prime}-\beta_{i^{\prime} j^{\prime}}^{(\hat{t}+1)}, \forall\left(i^{\prime}, j^{\prime}\right) \neq(i, j)\right\} \\
i=1, \cdots, N_{1}, j=1, \cdots, N_{2} \\
A_{i}^{(\hat{t}+1)}=\cup_{j} A_{i j}^{(\hat{t}+1)}, i=1, \cdots, N_{1} .
\end{gathered}
$$

Step 3 Given $\left\{z_{i}^{(t+1)}, i=1, \cdots, N_{1}\right\},\left\{A_{i}^{(t+1)}, i=1, \cdots, N_{1}\right\}$, update the $\left\{z_{i j}^{(t+1)}, i=1, \cdots, N_{1}, j=\right.$ $\left.1, \cdots, N_{2}\right\},\left\{A_{i j}^{(t+1)}, 1 \leq i \leq N_{1}, 1 \leq j \leq N_{2}\right\}$ by performing the following steps iteratively. For simplicity, write $A_{i}$ and $z_{i}$ short for $A_{i}^{(t+1)}$ and $z_{i}^{(t+1)}$ respectively in the following sub-steps. 
1) Given $\left\{A_{i j}^{(\hat{t})}, 1 \leq i \leq N_{1}, 1 \leq j \leq N_{2}\right\}$, the optimal codeword vectors for minimizing $\bar{D}$ at enhancement layer are computed respectively by

$$
\begin{gathered}
z_{i j}^{(\hat{t}+1)}=\arg \min _{\hat{z} \in A_{i j}^{(\hat{t})}} k_{1} \int_{z \in A_{i j}^{(\hat{t})}}\|z-\hat{z}\|^{2} f(z) \mathrm{d} z+\frac{p_{e 1}}{N_{2}\left(N_{1}-1\right)}\|\hat{z}\|^{2}+k_{2} \int_{z \in A_{i}}\|z-\hat{z}\|^{2} f(z) \mathrm{d} z, \\
i=1, \cdots, N_{1}, j=1, \cdots, N_{2} .
\end{gathered}
$$

2) Given $\left\{z_{i j}^{(\hat{t}+1)}, i=1, \cdots, N_{1}, j=1, \cdots, N_{2}\right\}$, the optimal finest partition is achieved by

$$
\begin{array}{r}
A_{i j}^{(\hat{t}+1)}=\left\{z:\left|z-z_{i j}^{(\hat{t}+1)}\right|^{2} \leq\left|z-z_{i j^{\prime}}^{(\hat{t}+1)}\right|^{2}, \forall j^{\prime} \neq j\right\}, \\
i=1, \cdots, N_{1}, j=1, \cdots, N_{2},
\end{array}
$$

Step 4 If $\left|\bar{D}^{(t+1)}-\bar{D}^{(t)}\right|$ is less than some predefined threshold then $\mathrm{t}=\mathrm{t}+1$, go to Step 2; if not stop.

Remark 10. It is easy to see that the quantization output formulas (3.19) and (3.20) are obtained from the following constrained optimization problems

$$
\begin{gathered}
\min _{z_{i}, i=1, \cdots, N_{1}} \bar{D} \quad \text { subject to } z_{i} \in A_{i}, i=1, \cdots, N_{1} . \\
\min _{z_{i j}, i=1, \cdots, N_{1}, j=1, \cdots, N_{2}} \bar{D} \quad \text { subject to } z_{i j} \in A_{i j}, i=1, \cdots, N_{1}, j=1, \cdots, N_{2} .
\end{gathered}
$$

Then we take the formula (3.20) as an example, and the formula (3.19) could be handled in a similar way. The solution to this problem will be (3.3) only if there exists a point within $A_{i j}$ so that $\frac{\partial \bar{D}}{\partial z_{i j}}=0$. Note that the objective function in (3.20) is actually a convex function with respect to $\hat{z}$. Therefore, one can apply a standard method such as in [40] to solve this constrained convex optimization problem. In practise, however, one can further use the following simplified method to update $z_{i j}^{(\hat{t}+1)}$ in the corresponding step of controlled iterative algorithm: if

$$
\begin{aligned}
& k_{1} \int_{A_{i j}^{(\hat{t})}} z f(z) \mathrm{d} z+k_{2} \int_{A_{i}} z f(z) \mathrm{d} z \\
& k_{1} p\left(A_{i j}^{(\hat{t})}\right)+\frac{p_{e 1}}{N_{2}\left(N_{1}-1\right)}+k_{2} p\left(A_{i}\right)
\end{aligned}
$$

lies in $A_{i j}^{(\hat{t})}$, then update $z_{i j}^{(\hat{t}+1)}$ according to 3.17$)$; otherwise, compute the point at the intersection between the boundary of $A_{i j}^{(\hat{t})}$ and the line segment connecting $z_{i j}^{(\hat{t})}$ and

$$
\frac{k_{1} \int_{A_{i j}^{(\hat{t})}} z f(z) \mathrm{d} z+k_{2} \int_{A_{i}} z f(z) \mathrm{d} z}{k_{1} p\left(A_{i j}^{(\hat{t})}\right)+\frac{p_{e 1}}{N_{2}\left(N_{1}-1\right)}+k_{2} p\left(A_{i}\right)}
$$


and then take the point as $z_{i j}^{(\hat{t}+1)}$. Because we create an additional constrain for the optimization problem, the set of the available solutions of this new formula is a subset of that without regular constraint. Based on that, it is not hard to obtain that the optimal solution with regular constrain should be no better than that of original optimization problem. Thus, controlled iterative algorithm should not outperform greedy iterative algorithm and new iterative algorithm in theoretical analysis.

Note that both greedy iterative algorithm and controlled iterative algorithm converge in the sense that the sequences of $\bar{D}^{(t)}$ are nonincreasing as $t \rightarrow \infty$. At this point, however, it is not clear whether the sequences of the corresponding quantizers given by $\left\{A_{i j}^{(t)}, 1 \leq\right.$ $\left.i \leq N_{1}, 1 \leq j \leq N_{2}\right\},\left\{z_{i}^{(t)}, i=1, \cdots, N_{1}\right\},\left\{z_{i j}^{(t)}, i=1, \cdots, N_{1}, j=1, \cdots, N_{2}\right\}$ will also converge in general. Although Wu proved the convergence of Lloyd-Max algorithm in [41] by using finite state machine, it is really a much harder problem to analyze the convergence of multi-resolution quantization algorithm. As authors known, there is no reference which solves this convergence problem, so it is interesting to deal with this problem in the future.

Simulations have been conducted to compare the performance of quantizers designed by greedy iterative algorithm with that of quantizers designed by controlled iterative algorithm. Given a quantizer and average error probabilities $p_{e 1}, p_{e 2}$ and $p_{b}$, the weighted EED for random index assignment is computed using (2.11). All experiments have been carried out for one-dimensional case, and we will demonstrate the relationships between the weighted distortion and each error probability above when fixing the other variables.

Consider a one-dimensional Gaussian source with zero mean and unit variance. Figure 3.8 and Figure 3.9 show the performance of these two algorihtms. As shown in the simulation results, the weighted distortion of controlled iterative algorithm increases as either $p_{e 1}$ or $p_{b}$ grows up.

It is also shown that greedy iterative algorithm outperforms the controlled iterative algorithm slightly, which coincides with our theoretic analysis in fact. The gain of greedy iterative algorithm over controlled iterative algorithm goes up as $p_{e 1}$ increases and it is basically non-related with the number of MRVQ levels, i.e. $N_{1}$ and $N_{2}$ as demonstrated in simulations. 

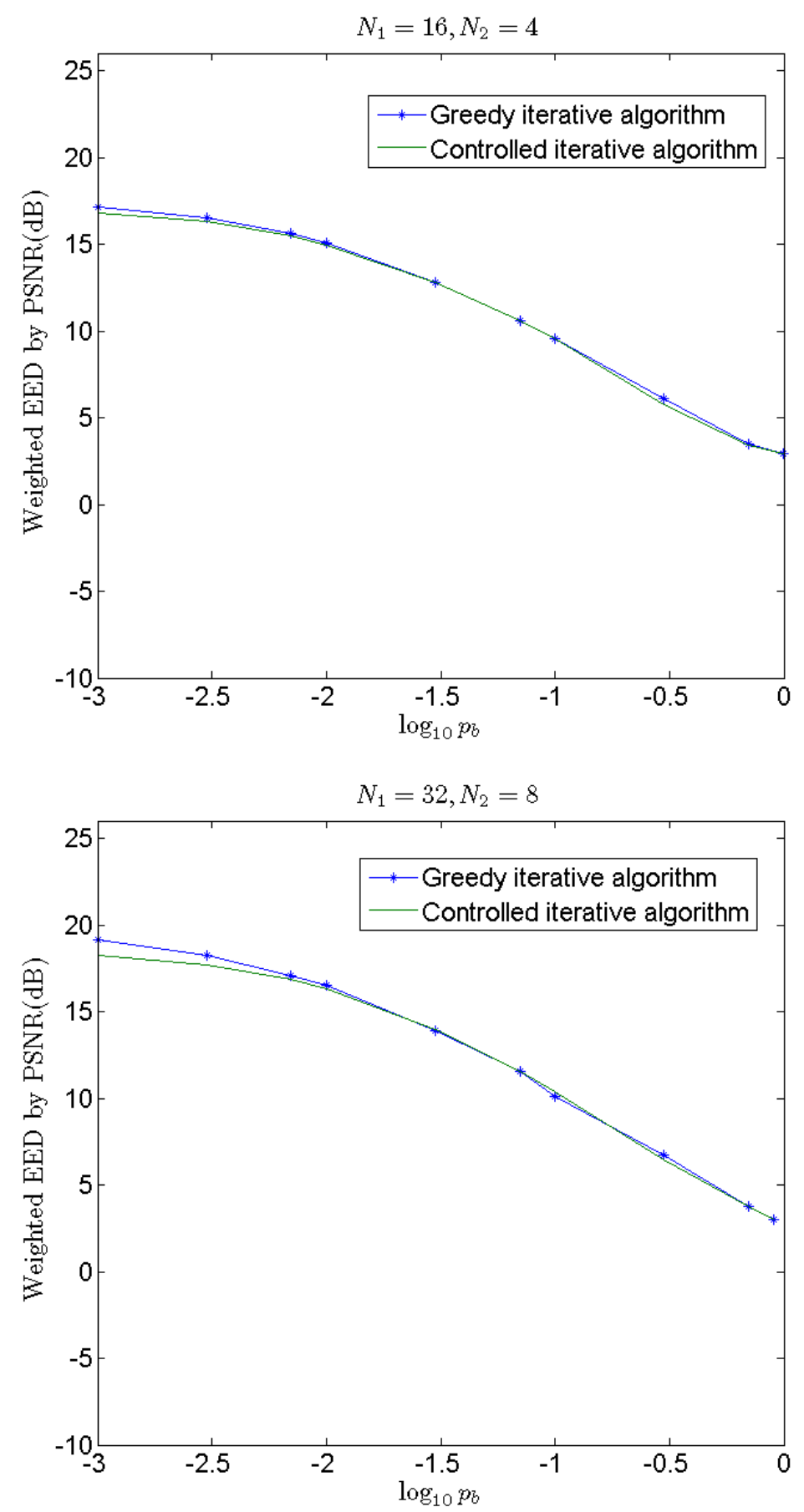

Figure 3.8: Comparison between greedy iterative algorithm and controlled iterative algorithm corresponding to various $p_{b}$ for a Gaussian source while fixing $p=0.5, p_{e 1}=0.01$ and $p_{e 2}=0.005$ 

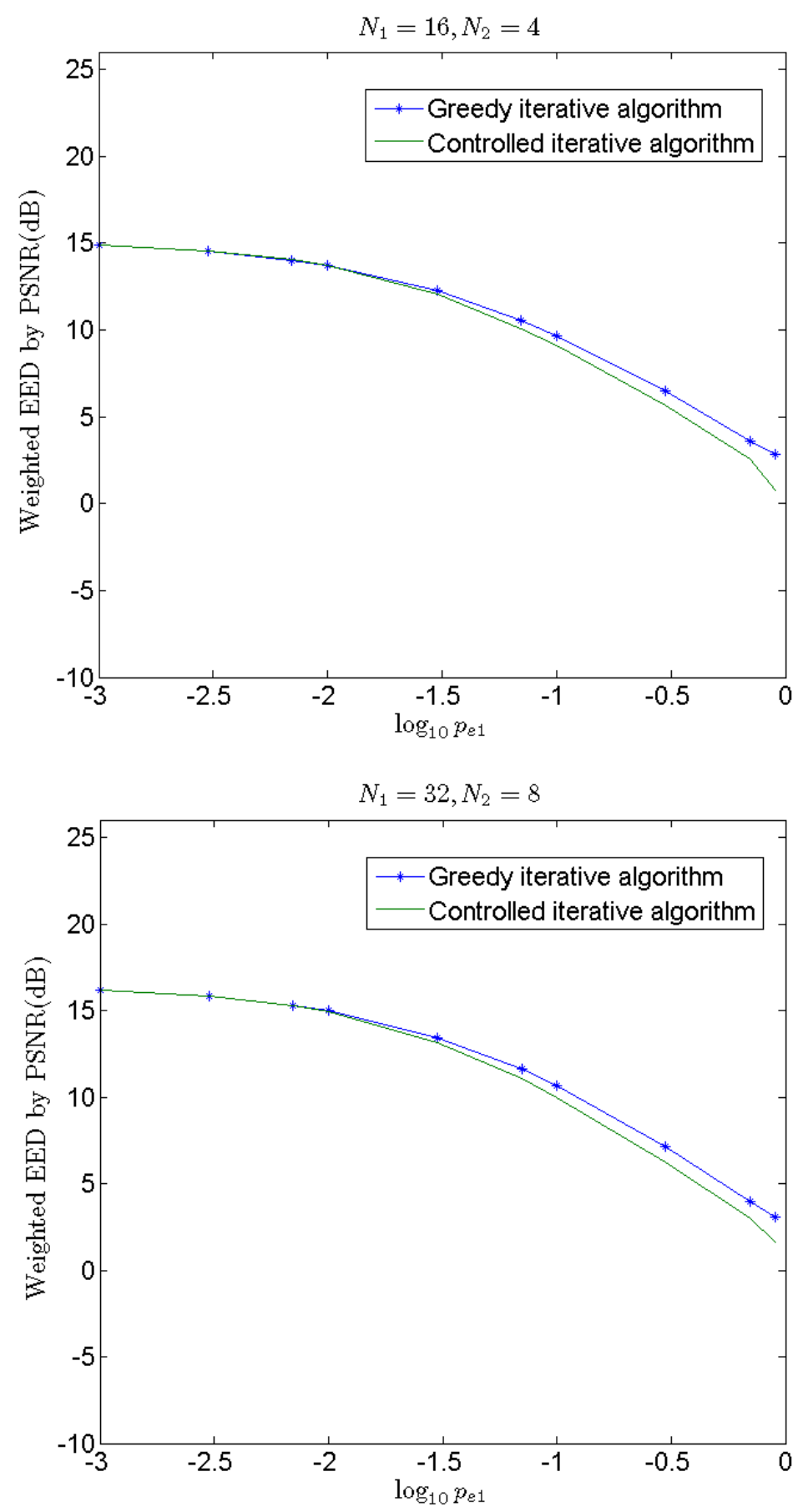

Figure 3.9: Comparison between greedy iterative algorithm and controlled iterative algorithm corresponding to various $p_{e 1}$ for a Gaussian source while fixing $p=0.5, p_{b}=0.02$ and $p_{e 2}=0.005$ 


\section{Chapter 4}

\section{The Asymptotic Minimum Weighted EED}

In this section, we study the asymptotic high rate behavior of the optimality criterion 2.11 and we will derive the infimum of the weighted EED over all possible quantizers.

For any $k$-dimensional 2-resolution quantizer $\mathrm{Q}$ with $N_{1} \times N_{2}$ levels, denote the average EEDs in Theorem 1 and the weighed EED in 2.11 by $D_{b}^{\Pi}(Q), D_{e}^{\Pi}(Q)$ and $\bar{D}(Q)$ respectively, i.e.,

$$
\bar{D}(Q)=p D_{e}^{\Pi}(Q)+(1-p) D_{b}^{\Pi}(Q)
$$

Define $\bar{D}\left(N_{1}, N_{2}\right) \triangleq \inf _{Q} \bar{D}(Q)$ where the infimum is taken over all $k$-dimensional $N_{1} \times$ $N_{2}$-level quantizers $Q$. Then the following is asymptotic result:

Theorem 3. Given the average error probability $p_{e 1}, p_{b}$ for the first channel and the second channel respectively and the proportion $p$,

$$
\lim _{N_{1} \rightarrow \infty} \bar{D}\left(N_{1}, N_{2}\right)=p \cdot p_{e 1} \sigma^{2}+(1-p) p_{b} \sigma^{2}
$$

Proof. In view of (2.6), 2.5 and 2.11, it is not hard to get,

$$
\lim _{N_{1} \rightarrow \infty} \inf \bar{D}\left(N_{1}, N_{2}\right) \geq p \cdot p_{e 1} \sigma^{2}+(1-p) p_{b} \sigma^{2} .
$$

Thus, it suffices to prove

$$
\lim _{N_{1} \rightarrow \infty} \sup \bar{D}\left(N_{1}, N_{2}\right) \leq p \cdot p_{e 1} \sigma^{2}+(1-p) p_{b} \sigma^{2}
$$


To this end, we consider random quantization. Let $\lambda_{i}(z), i \in\{1,2\}$ be a positive density function over the $k$-dimensional Euclidean space $\Lambda$ satisfying

$$
\begin{gathered}
\int_{\Lambda} \lambda_{i}(z) \mathrm{d} z=1 \\
\int_{\Lambda}|z|^{2} \lambda_{i}(z) \mathrm{d} z<\infty .
\end{gathered}
$$

Randomly pick code vectors $z_{i}$ with the common density function $\lambda_{1}(z)$ and $z_{i j}$ with the common density function $\lambda_{2}(z), 1 \leq i \leq N_{1}, 1 \leq j \leq N_{2}$, where all of them are independent. For convenience, we write $\left\{z_{1}, \cdots, z_{N_{1}}\right\}$ as $Z^{N_{1}}$ and $\left\{z_{i j}, 1 \leq i \leq N_{1}, 1 \leq j \leq N_{2}\right\}$ as $Z^{N_{1} N_{2}}$. $Z^{N_{1}}$ and $Z^{N_{1} N_{2}}$ decide a random quantizer which maps $z$ to $z_{i}$ and $z_{i j}$ for each resolution if $F\left(z, z_{i}, z_{i j}\right)$ is minimum with respect to $(\mathrm{i}, \mathrm{j})$, where $F\left(z, z_{i}, z_{i j}\right)$ is defined in (3.6),

$$
F\left(z, z_{i}, z_{i j}\right)=\frac{p}{k}\left(k_{1}\left\|z-z_{i j}\right\|^{2}+k_{2} \sum_{\hat{j}=1}^{N_{2}}\left\|z-z_{i j}\right\|^{2}\right)+\frac{(1-p) k_{3}}{k}\left\|z-z_{i}\right\|^{2} .
$$

From the definition of $\bar{D}\left(N_{1}, N_{2}\right)$ it follows that, for any $Z^{N_{1}}, Z^{N_{1} N_{2}}$

$$
\begin{aligned}
\bar{D}\left(N_{1}, N_{2}\right) \leq & \frac{1}{k} \int_{\Lambda} \min _{i, j} F\left(z, z_{i}, z_{i j}\right) f(z) \mathrm{d} z+\frac{p N_{1} p_{e 1}}{N_{1}-1}\left(\sigma^{2}+\frac{1}{k N_{1} N_{2}} \sum_{i=1}^{N_{1}} \sum_{j=1}^{N_{2}}\left\|z_{i j}\right\|^{2}\right) \\
& +\frac{(1-p) N_{1} p_{b}}{N_{1}-1}\left(\sigma^{2}+\frac{1}{k N_{1}} \sum_{i=1}^{N_{1}}\left\|z_{i}\right\|^{2}\right)
\end{aligned}
$$

that implies that

$$
\begin{aligned}
\bar{D}\left(N_{1}, N_{2}\right) \leq & \frac{1}{k} \int_{\Lambda} E\left[\min _{i, j} F\left(z, z_{i}, z_{i j}\right)\right] f(z) \mathrm{d} z+\frac{p N_{1} p_{e 1}}{N_{1}-1}\left(\sigma^{2}+\frac{1}{k} E\left[\left\|z_{11}\right\|^{2}\right]\right) \\
& +\frac{(1-p) N_{1} p_{b}}{N_{1}-1}\left(\sigma^{2}+\frac{1}{k} E\left[\left\|z_{1}\right\|^{2}\right]\right)
\end{aligned}
$$

where $E$ denotes the expectation with respect to $Z^{N_{1}}$ and $Z^{N_{1} N_{2}}$. We next show that for any fixed $z \in \Lambda$,

$$
\lim _{N_{1} \rightarrow \infty} E\left[\min _{i j} F\left(z, z_{i}, z_{i j}\right)\right]=0
$$


To this end, define for any $\varepsilon>0$,

$$
p(z, \varepsilon) \triangleq \operatorname{Pr}\left\{\min _{j} F\left(z, z_{1}, z_{1 j}\right)<\varepsilon^{2}\right\}
$$

Then we have,

$$
\operatorname{Pr}\left\{\min _{i j} F\left(z, z_{i}, z_{i j}\right)>\varepsilon^{2}\right\}=(1-p(z, \varepsilon))^{N_{1}}
$$

which goes to 0 exponentially fast as infinity. Since

$$
\min _{i j} F\left(z, z_{i}, z_{i j}\right) \leq \min _{j} F\left(z, z_{1}, z_{1 j}\right)
$$

and also as $N_{1}$ is large enough, $\min _{i j} F\left(z, z_{i}, z_{i j}\right) \geq 0$, it is not hard to verify that,

$$
E\left[\min _{i j} F\left(z, z_{i}, z_{i j}\right)\right] \leq \varepsilon^{2}+(1-p(z, \varepsilon))^{N_{1}} E\left[\min _{i j} F\left(z, z_{i}, z_{i j}\right) \mid \min _{i j} F\left(z, z_{i}, z_{i j}\right)>\varepsilon^{2}\right]
$$

where $E[\cdot \mid \cdot]$ denotes the conditional expectation. Letting $N_{1} \rightarrow \infty$ and then $\varepsilon \rightarrow 0$ yields (4.6).

Go back to (4.5). In view of (4.6) and the dominated convergence theorem, letting $N_{1} \rightarrow \infty$ in 4.5 implies that

$$
\begin{aligned}
\lim _{N_{1} \rightarrow \infty} \sup \bar{D}\left(N_{1}, N_{2}\right) & \leq p \cdot p_{e 1} \sigma^{2}+(1-p) p_{b} \sigma^{2}+\frac{p \cdot p_{e 1}}{k} E\left[\left|Z_{11}\right|^{2}\right]+\frac{p \cdot p_{b}}{k} E\left[\left|Z_{1}\right|^{2}\right] \\
& =p \cdot p_{e 1} \sigma^{2}+(1-p) p_{b} \sigma^{2}+\frac{p \cdot p_{e 1}}{k} \int_{\Lambda}|z|^{2} \lambda_{2}(z) \mathrm{d} z+\frac{p \cdot p_{b}}{k} \int_{\Lambda}|z|^{2} \lambda_{1}(z) \mathrm{d} z
\end{aligned}
$$

Letting $\lambda_{1}(z)$ and $\lambda_{2}(z)$ approach the delta function yields 4.1.

Remark 11. The proof of Theorem 3 illustrates the optimal quantizer design for a given broadcast channel. When the quantization rate at the base layer is high, there will be a sufficiently large number of code vectors in each layer scattering over the whole space so that the integral of $F\left(z, z_{i}, z_{i j}\right)$ approaches zero, on the other hand most code vectors for each resolution will be in a small region around the source mean to make the energy of each set of code vectors arbitrary small in order to achieve $\bar{D}\left(N_{1}, N_{2}\right)$. Consequently, from the asymptotic analysis we suppose that for any noise channels the code vectors for each resolution quantization will approach the delta-like pattern when $N_{1}$ is large enough. Note that the asymptotic performance is not dependent on $p_{e 2}$, which coincides the results in Figure 3.5 to some degree. Besides, only if $N_{1}$ is sufficiently large, for any $N_{2}$ this conclusion always maintains true.

Now we will compare the asymptotic performance given in Theorem 3 with that of separate $M R V Q$ design without reference to the channel noise. In the separate design, 
the quantization code vectors and partitions are designed independently from the channel conditions so as to minimize the quantization distortion. Let $Q_{M R}^{s e p}$ denote the optimal $M R V Q$ without channel noise.

$A s N_{1} \rightarrow \infty, p \bar{D}_{Q_{e}}\left(Q_{M R}^{s e p}\right)+(1-p) \bar{D}_{Q_{b}}\left(Q_{M R}^{\text {sep }}\right)$ goes to 0. Therefore, for sufficiently large $N_{1}$,

$$
\bar{D}\left(Q_{M R}^{s e p}\right) \geq p \cdot p_{e 1} \sigma^{2}+(1-p) p_{b} \sigma^{2}+p \cdot p_{e 1} S_{Q_{e}}+(1-p) p_{b} S_{Q_{b}}
$$

In comparison with Theorem 3, we have another two terms in (4.7). For the optimal $M R V Q$ without channel noise, the last two terms cannot be ignored when minimizing the distortion of quantization only. So the gain of joint $M R V Q$ design with channel conditions over separate MRVQ without reference to the channel noise is quite significant. 


\section{Chapter 5}

\section{Conclusion and Future Work}

\subsection{Conclusion}

In a communication system that doesn't allow delay, Shannon's classical separation result is on longer valid. In such cases where source code dimension or channel code block length is not arbitrarily long, a joint source and channel code design could be used to improve communication performance. Especially in a multiuser system, joint source channel coding attracts more and more interests due to its adaptivity and consistency. Multiresolution source codes are useful in a multicast system, where the available communication rate may vary as a function of network traffic and physical location. As far as the author knows, there is seldom work on multiresolution source coding over multiuser networks. In this thesis, we present the design and analysis of the optimal multiresolution vector quantization in conjunction with a broadcast channel over which the coded scalable bit stream would be transmitted. The main results of this thesis are elaborated as follows.

Given a broadcast system with a multiresolution vector quantizer, a random index assignment, and a coded broadcast channel, we have established closed formulas for the average end-to-end distortion at each receiver of the system. Based on these formulas, we analyze the intrinsic structure of end-to-end distortion in a communication system. Two necessary conditions for minimizing a weighted end-to-end distortion are derived, and further we propose an greedy iterative algorithm for jointly designing MRVQ with channel conditions. Experiments are conducted to show that the MRVQ designed by the proposed algorithm significantly outperforms conventional MRVQ designed without channel information. In addition, we explore another two novel algorithms for MRVQ design over broadcast channels companied with interesting simulation results. Finally, the asymptotic performance of the weighted EED for the optimal joint MRVQ is analyzed.

Compared with the noisy MRVQ design in [36], the greedy iterative algorithm does 
not need the complete knowledge of the channel (i.e., all transitional probabilities from the inputs of the channel to the outputs of the channel) - it depends on the channel only through several types of average channel error probabilities. Our proposed method is more efficient computationally for multiresolution quantization design and more robust against channel mismatch. As such, our proposed algorithm is more attractive both in wireless

multicast communications where the transitional probabilities of the channel fluctuate and in applications where the complete knowledge of the channel is unknown.

\subsection{Future work}

The results of this thesis are just starting point towards practical scheme of multiresolution quantization design over broadcast channels. Several critical problems have to be answered before an efficient implementation is found.

1 As the proposed design is just one of approaches to design practical MRVQ over broadcast channels, are there any other ways which are more efficient and easier to design and converge to the globally optimum?

2 If utilizing cyclic redundancy check in our joint source channel coding design, would it reduce distortion resulting from channel noise and improve the performance?

3 How to extend the theoretic joint MRVQ design in this thesis to the design for audio and video signals? In particular, it will be more interesting if the design are compatible with industrial standards like MP3 and H.264 to achieve efficient tradeoffs among complexity, distortion and robustness.

4 Could we optimize both source coding and channel coding simultaneously in a multicast system, and find the best rate allocation between source rate and channel coding rate? 


\section{References}

[1] W. H. R. Equitz and T. M. Cover, "Successive refinement of information," IEEE Trans. on Inform. Theory, Vol. 37, pp. 269-275, May. 1991. 21

[2] M. Effros, "Distortion-rate bounds for fixed- and variable-rate multiresolution source codes," IEEE Trans. on Inform. Theory, Vol. 45, pp. 1887-1910, Sept. 1999. 4, 21

[3] S. Dumitrescu and X. Wu, "Algorithms for optimal multiresolution quantization," Journal on Algorithms, 50:1-2, 2004. 4

[4] M. Effros and D. Dugatkin, "Multiresolution Vector Quantization" IEEE Trans. on Inform. Theory, Vol. 50, No. 12 pp. 3130-3145 Dec. 2004. 4, 20, 21, 24, 25

[5] M. Effros, "Practical multi-resolution source coding: TSVQ revisited," in Proc. Data Compression Conf., Snowbird, UT, Mar. 1998, pp. 5362. 4

[6] C. E. Shannon, "Coding theorems for a discrete source with a fidelity criterion," in IRE Nat. Conv. Rec, Part 4, 1959, pp. 142163. 5

[7] J. W. Modestino and D. G. Daut, "Combined source-channel coding of images," IEEE Trans. Commun., vol. COM-27, pp. 16441659, Nov. 1979. 5

[8] K.-P. Ho and J. M. Kahn, "Transmission of analog signals using multicarrier modulation: A combined source-channel coding approach," IEEE Trans. Commun., vol. 44, pp. 14321443, Nov. 1996. 5

[9] A. Goldsmith and M. Effros, "Joint Design of Fixed-Rate Source Codes and Multiresolution Channel Codes," IEEE Trans. on Communications, Vol.46, No.10, Oct. 1998 6]

[10] N. Farvardin, "A study of vector quantization for noisy channels," IEEE Trans. Inform. Theory, vol. 36, pp. 799809, July 1990.

[11] K. Zeger and A. Gersho, "Pseudo-Gray coding," IEEE Trans. Commun., vol. 38, pp. 21472158, Dec. 1990. 
[12] S. Gadkari and K. Rose, "Vector quantization with transmission energy allocation for time-varying channels," IEEE Trans. Commun., VOL. 47, NO. 1, JANUARY 19996

[13] N. Farvardin and V. Vaishampayan, "On the performance and complexity of channeloptimized vector quantizers," IEEE Trans. Inform. Theory, vol. 37, pp. 155160, Jan. 1991. 6]

[14] H. Kumazawa, M. Kasahara, and T. Namekawa, "A construction of vector quantizers for noisy channels," Electron. Eng. in Japan, vol. 64B, no. 4, pp. 3947, 1984. 6, 7]

[15] N. Farvardin and V. Vaishampayan, "Optimal quantizer design for noisy channels: An approach to combined source-channel coding," IEEE Trans. Inform. Theory, vol. 33, pp. 827838, Nov. 1987. 6

[16] M. Effros, "Robustness to channel variation in source coding for transmission across noisy channels," in Proc. IEEE ICASSP, Apr. 1997, pp. 29612964.

[17] V. A. Vaishampayan and N. Farvardin, "Joint design of block source codes and modulation signal sets," IEEE Trans. Inform. Theory, vol. 38, pp. 12301248, July 1992. 6

[18] K.-P. Ho and J. M. Kahn, "Combined source-channel coding using channel-optimized quantizer and multicarrier modulation," in Proc. IEEE ICC96, June 1996, pp. 13231327. 6

[19] A. Gersho and R. M. Gray, "Vector Quantization and Signal Compression." New York: Kluwer Academic, 1992. 6

[20] J. Dunham and R. M. Gray, "Joint source and noisy channel trellis encoding," IEEE Trans. Inf. Theory, vol. IT-27, no. 4, pp. 516519, Jul. 1981. 7

[21] R. Hagen and P. Hedelin, "Robust vector quantization by a linear mapping of a block code," IEEE Trans. Inf. Theory, vol. 45, no. 1, pp. 200218, Jan. 1999. 7

[22] B. Farber and K. Zeger, "Quantizers with uniform encoders and channel optimized decoders," IEEE Trans. Inf. Theory, vol. 50, no. 1, pp. 6277, Jan. 2004. 7

[23] B.Farber and K. Zeger, "Quantizers With Uniform Decoders and Channel Optimized Encoders," IEEE Trans. on Information Theory, Vol.52, No.2, Oct. 20067

[24] T. S. Huang, "Optimum Binary Code," MIT Res. Lab. Electron, Cambridge, MA, Quart. Progr. Rep. 82, 1966. 7

[25] T. R. Crimmins, H. M. Horwitz, C. J. Palermo, and R. V. Palermo, "Minimization of mean-square error for data transmitted via group codes," IEEE Trans. Inf. Theory, vol. IT-15, no. 1, pp. 7278, Jan. 1969. 7 
[26] A. Mhes and K. Zeger, "Binary lattice vector quantization with linear block codes and affine index assignments," IEEE Trans. Inf. Theory, vol. 44, no. 1, pp. 7994, Jan. 1998. 7

[27] A. Mhes and K. Zeger, "Randomly chosen index assignments are asymptotically bad for uniform sources," IEEE Trans. Inf. Theory, vol. 45, no. 2, pp. 788794 Mar. 1999. 7

[28] S. W. McLaughlin, D. L. Neuhoff, and J. J. Ashley, "Optimal binary index assignments for a class of equiprobable scalar and vector quantizers," IEEE Trans. Inf. Theory, vol. 41, no. 6, pp. 20312037, Nov. 1995. 7

[29] R. Gallager, "Information Theory and Reliable Communication." New York: Wiley, 1968

[30] D. Tse and Viswanath, "Fundamentals of wireless communication." Cambridge: Cambridge University Press. 14

[31] T. M. Cover and J. Thomas, "Elements of Information theory." Wiley Series in Telecommunications. 13, 14, 15

[32] K. Zeger, and V. Manzella, "Asymptotic bounds on optimal noisy channel quantization via random coding," IEEE Trans. on Inform. Theory, Vol. 40, No. 6, pp.1926-1938, Nov. 1994. 8, 30,

[33] X. Yu, H. Wang, and E.-H. Yang, "Optimal quantization for noisy channels with random index assignment," Proc. of the 2008 IEEE Intern. Symp. Inform. Theory, Toronto, Canada, July 6-11, pp. 2727-2731, 2008. 25

[34] X. Yu, H. Q. Wang, and E.H. Yang, "Design and analysis of optimal noisy channel quantization with random index assiangment," IEEE Trans. on Inform. Theory, submitted for publication. 19, 25

[35] F. Teng, E.-H. Yang, and X. Yu, "Design and analysis of optimal multiresolution quantization for broadcast channels with random index assignment," in preparation for submission to IEEE Trans. on Inform. Theory.

[36] I. Kozintsev and K. Ramchandran, "Multiresolution joint source-channel coding using embedded constellations for power constrained time varying channels," in Proc. ICASSP, 1996, pp. 2343-2346. 6, 10, 35, 51

[37] H. Brunk and N. Farvardin, "Fixed-rate successively refinable scalar quantizers," Proc. Data Compression Conf., Snowbird, UT, Apr. 1996, pp. 250-259. 21, 24, 25, 29 
[38] S. Lloyd, "Least squares quantization in PCM, IEEE Trans. on Information Theory, Vol. IT-28, No.2, pp.129-137, Mar. 1982. 28

[39] J. Max, "Quantizing for minimum distortion, IRE Transactions on Information Theory, Vol. IT-6, pp.7-12, Mar. 1960. 28

[40] S. Boyd, L. Vandenberghe, "Convex Optimization," Cambridge University Press, 2006. 43

[41] X. Wu, "On convergence of Lloyds method I," IEEE Trans. on Information Theory, Vol. 38, No.1, pp.171-174, Jan. 1992. 44 